\title{
Modelling and planning reliable wireless sensor networks based on multi-objective optimization genetic algorithm with changeable length
}

\author{
Danping He - Gabriel Mujica • Jorge Portilla • \\ Teresa Riesgo
}

\begin{abstract}
Wireless sensor networks (WSN) have shown their potentials in various applications, which bring a lot of benefits to users from different working areas. However, due to the diversity of the deployed environments and resource constraints, it is difficult to predict the performance of a topology. Besides the connectivity, coverage, cost, network longevity and service quality should all be considered during the planning procedure. Therefore, efficiently planning a reliable WSN is a challenging task, which requires designers coping with comprehensive and interdisciplinary knowledge. A WSN planning method is proposed in this work to tackle the above mentioned challenges and efficiently deploying reliable WSNs. First of all, the above mentioned metrics are modeled more comprehensively and practically compared with other works. Especially 3D ray tracing method is used to model the radio link and sensing signal, which are sensitive to the obstruction of obstacles; network routing is constructed by using AODV protocol; the network longevity, packet delay and packet drop rate are obtained via simulating practical events in WSNet simulator, which to the best of our knowledge, is the first time that network simulator is involved in a planning algorithm. Moreover, a multi-objective optimization algorithm is developed to cater for the characteristics of WSNs. Network size is changeable during evolu-
\end{abstract}


tion, meanwhile the crossovers and mutations are limited by certain constraints to eliminate invalid modifications and improve the computation efficiency. The capability of providing multiple optimized solutions simultaneously allows users making their own decisions, and the results are more comprehensive optimized compared with other state-of-the-art algorithms. Practical WSN deployments are also realized for both indoor and outdoor environments and the measurements coincident well with the generated optimized topologies, which prove the efficiency and reliability of the proposed algorithm.

Keywords Efficient planning method - Measurement of WSN · Modeling of WSN · Multi-objective optimization · NSGA-II

\section{Introduction}

Recent years have witnessed an increased interest in the use of wireless sensor networks (WSNs) in various applications such as environmental monitoring, space exploration, factory automation, habitat tracking, secure surveillance, and battlefield surveillance. This technology has brought a lot of benefits to the users from both research and industrial areas. In WSNs, miniaturized sensor nodes are deployed to operate autonomously in different types of environments. Sensor networks may consist of different types of sensors such as seismic, visual, infrared, acoustic and so on. In addition to the ability to probe its surroundings, each sensor node has an onboard radio to communicate with other nodes through wireless communication protocols such as $\mathrm{ZigBee}^{T M}$ (2007), Bluetooth $^{T M}$ (1998) and Ultra-wideband (UWB) (2009) among others. The sensed data are collected and sent to a base station directly or via multiple hops depending on the network topology and routing protocols. For many setups, it is envisioned that WSNs will consist of tens to hundreds of nodes that operate on small batteries. A sensor stops working when it runs out of energy and results in a damage in the WSN structure.

The major challenge in designing WSNs is the support of various application requirements while coping with the computation, energy, communication, sensing and cost constraints. Careful node placement can be a very effective optimization means for achieving the desired design goals. However, optimal node placement has been proven to be NP-Hard for most of the formulations of node deployment (Efrat et al. 2004; Cheng et al. 2008; Poduri et al. 2006). Several works are developed to tackle the efficiency of planning algorithms: Misra et al. (2010) concern the relay node placement problem for WSNs in the aim of placing a minimum number of relay nodes into a WSN to meet certain connectivity or survivability requirements. Relay nodes can only be placed at a set of candidate locations and they discuss the computational complexity and present a framework of polynomial time $O(1)$-approximation algorithms with small approximation ratios. Numerical results show that their approximation algorithms can produce solutions very close to optimal solutions. Shams et al. (2008) propose an approximation algorithm that runs in $O\left(n^{2}\right)$ time complexity, it targets to find a feasible solution for minimizing number of relay nodes in such a fashion that each sensor node must have at least one relay node within its one hop distance. 
Lee and Lee (2013) presents a polynomial-time relay node placement algorithms using Minimum Steiner tree on convex hull.

Beyond the complexity control, most planning methods focus on modeling important parameters that have strong impacts on the network performance, and on optimizing topology based on the modeled metrics. In this work, only those methods with 3D calculation ability are investigated.

The 3D indoor planning heuristic (LowCost) proposed by Kouakou et al. (2010), to the best of our knowledge, is the first indoor 3D WSN deploy heuristic that considers impacts of obstacles. It consists of two steps: Provided a 3D indoor environment model with furniture and obstacles recorded, the first step calculates the coverage to deployment cost ratio for all the candidate points in the deployable area. Sensor nodes are iteratively put at the point with the maximum coverage to deployment cost ratio, so that the target region is covered with the minimum sensor node cost after this step. Then the connectivity of the deployed nodes is checked in the second step. The authors consider two options to satisfy the connectivity of WSN, the prior one is realized by moving the unconnected node towards the closest connected node without influencing the sensing coverage of the first step; otherwise, if the preferential option is not applicable, extra sensor nodes will be added along the line between the unconnected node and the closest connected node. Note that despite this approach manages to cover the sensing area with the "minimum cost", the connectivity of the WSN is ensured by simply moving or placing extra nodes without carefully selecting optimal positions to decrease the hardware cost, improve the link quality or prolong the network lifetime. Moreover, although the modelling of the sensing signal considers obstacles, the radio propagation model is too simple because the communication links are established only between line-of-sight nodes, which is obviously not true in the real-world propagation.

The MOGA algorithm (Jourdan and de Weck 2004) employs multi-objective genetic algorithm, which is proved to be efficient in solving NP-hard problem, to evolve the decision. Topology solution for the same network varies at different runs, which provides more options than the deterministic approach of LowCost. However, it focuses on maximizing the sensing coverage and prolonging the network lifetime with a predetermined number of nodes, as a result the hardware cost can not be optimized. Moreover, the modeling of radio signal and sensing signal are based on ideal disc model thus it is not environmental sensitive.

The authors of Shams et al. (2008) propose an approximation algorithm to find a feasible solution for relay node placement to deploy a minimum set of relay nodes in such a fashion that each sensor node must have at least one relay node within its one hop distance and all deployed relay nodes eventually form a connected network among themselves including one or more base-stations. The work reveals an approximation algorithm that runs in $O\left(n^{2}\right)$ time complexity, to find a feasible solution for the above challenge.

The work in Kim et al. (2007) proposes multiple-objective metric for base station placement in WSNs to fairly increase various properties. It considers four different metrics for base station placement in WSNs. First, the ratio of sensor nodes which can communicate with a base station via either single-hop or multi-hop represents the coverage of sensor nodes. Second, the average ratio of connected sensor nodes after the failure of base stations represents the fault tolerance of a network. Third, the average 
distance between sensor nodes and their nearest base station represents the energy consumption of a network. However, as discussed before, not only the distance but also the obstacles lead to attenuation of the received signal strength (RSS). Moreover, more energy is consumed at nodes with larger degree, as a result the energy consumption is not practically modelled by this work. Fourth, the standard deviation of the degree of base stations represents the average delay of a network due to congestions. The limitation of this algorithm is that sensor nodes should be pre-located by designers, which neither guarantees the sensing coverage without expert experience nor allows optimizing the hardware cost for WSN.

The coverage problem of WSNs for the rolling terrains is studied in Liu and Ma (2012) to derive the general expression of the expected coverage ratio for regular terrains and irregular terrains.

Huang et al. (2008) develop a tool that integrates a 3D indoor deployment heuristic together with NS-2 simulator to assist designers deploying and analyzing the performance of networks. They propose a heuristic that minimizes hardware cost while satisfying requirements on coverage and connectivity. The network topology is constraint to the type of cluster tree and three different devices are provided: the coordinator, router and sensor. Sensors can only communicate with routers and coordinator. The heuristic considers radiation pattern of antenna as well as the effects of obstacles by using an accurate ray-tracing algorithm. Once the topology is generated, the integrated NS-2 simulator is driven to simulate the packet drop rate and latency, and the results are demonstrated to users. The merit of this method is the integration of an authorized network simulator to evaluate the performance of generated topologies, which provides a much more practical implication on packet delivery performance to designers. However, as the evaluation from NS-2 has no contribution on improving the generated topology, the proposed deployment heuristic should be run several times so that by a certain chance, designers can observe a satisfied solution with low cost, low drop rate and latency. The user interface allows users to prosecute many configurations including map, node properties, topology constraints and environment types. The generated topology can be shown and results evaluated by NS-2 are reported on the interface.

A method for deploying relay node and sink node for indoor environment is proposed by Guinard et al. (2011) and McGibney et al. (2011), the tool allows users defining the node demand zones, power source, sensing interval and transmission delay. By encapsulating those metrics into a complete requirements model, the tool optimizes the infrastructure of WSN and maximizes the utility function, which provides a normalized equation that observes the coverage, link quality, lifetime and infrastructure cost. The lifetime $(L)$ of sensor node is considered in that work and is modelled by (2), The electric charge of a sensor node $E C$, expressed in $\mathrm{mAh}$, is calculated according to (1) where $I_{a}$ and $I_{s}$ are the power consumption in active state and sleep state respectively. $t_{a}$ and $t_{s}$ represents their time durations in a node interval. The current capacity of the battery $C C$ is expressed in $\mathrm{mAh}$. Figures 1 and 2 are examples of generated solutions for single-hop and multihop topologies respectively. The model of lifetime only represents a coarse estimation and only on sensor nodes, moreover the authors did not consider the modeling of packet delivery ability to ensure a more reliable WSN. 


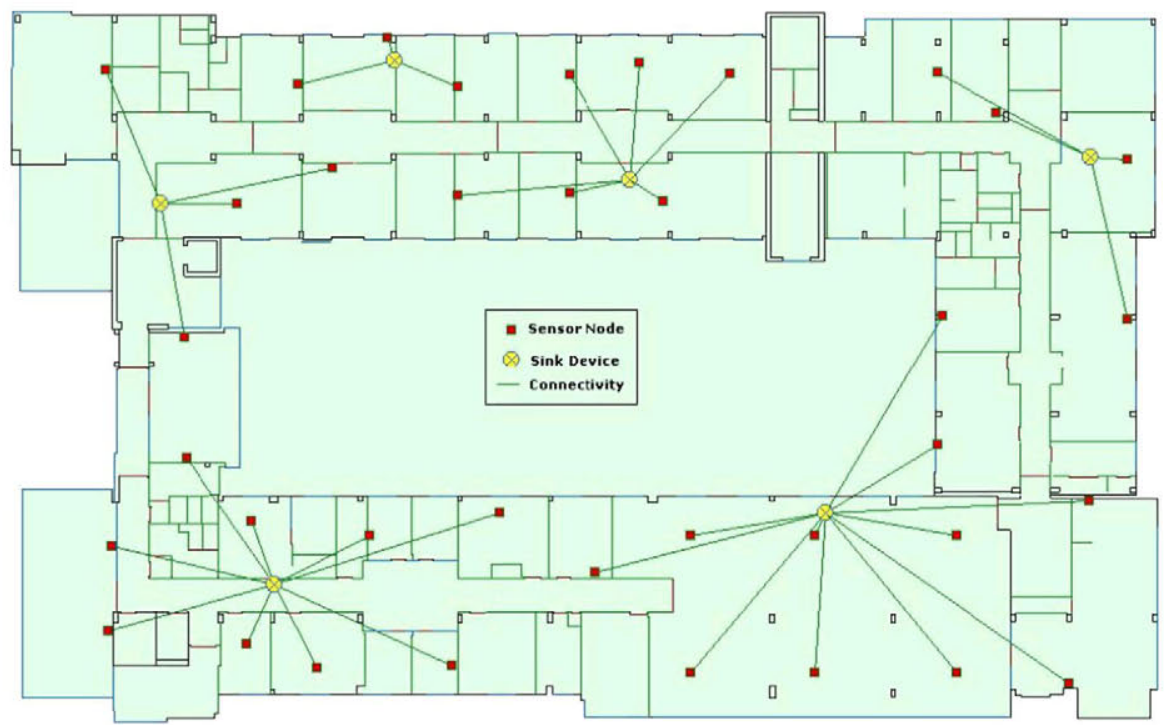

Fig. 1 Demonstration single-hop solution of work in Guinard et al. (2011)

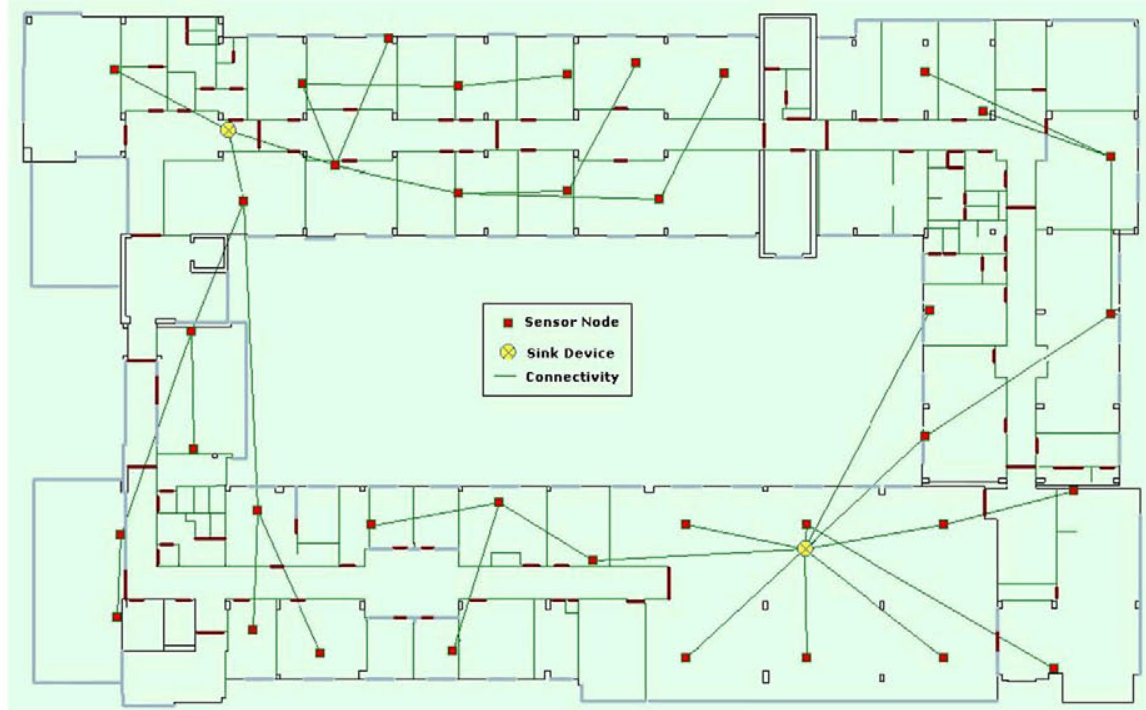

Fig. 2 Demonstration multi-hop solution of work in Guinard et al. (2011)

$$
\begin{aligned}
E C & =\frac{3600}{t_{a}+t_{s}} \times\left(t_{a} \times I_{a}+t_{s} \times I_{s}\right) \\
L & =\frac{C C}{E C}
\end{aligned}
$$


There are works focused on tackling characterization on very specific metrics, such as Akbarzadeh et al. (2013) develop a probabilistic sensing model for sensors with line-of-sight-based coverage (e.g. cameras). The probabilistic sensing model takes into consideration the sensing capacity probability as well as critical environmental factors such as terrain topography. Sensor deployment in network-structured environments is studied by Xiong et al. (2012) and it aims to achieve k-coverage while minimizing the number of sensor nodes. The coverage problem of WSNs for the rolling terrains is studied by Liu and Ma (2012) to derive the general expression of the expected coverage ratio for regular terrains and irregular terrains.

The aforementioned algorithms and tools, to some extent, have serious limitations for practical use. From the aspect of metric modelling, only a few of them tackles the 3D deployment issues (Kouakou et al. 2010; Guinard et al. 2011; McGibney et al. 2011). Even fewer methods model the sensing coverage and radio propagation by considering realistic scenarios where obstacles present. None of the aforementioned algorithms modeled the network longevity properly and practically, unilateral and unrealistic formulations are often employed. Moreover, the optimization targets are often one-sided in the introduced works. Without comprehensive evaluation on the important metrics, the performance of WSNs can not be entirely optimized.

An efficient WSN planning algorithm is proposed in this paper to tackle the above mentioned challenges and efficiently assist designers on deploying reliable WSNs. This work contributes on the following aspects:

- Comprehensive metrics are considered. This work considers connectivity, sensing coverage, cost, lifetime, packet latency and packet drop rate, which to the best of our knowledge, is the most comprehensive evaluation scheme for analyzing the performance of WSN.

- Practical metrics modelling by integrating network simulator. The connectivity and sensing coverage are modelled in assistance of 3D ray-tracing method, which is sensitive to the existence of obstacles; hardware cost refers to the number of devices as well as their types; routes of network is constructed by using Ad hoc on-demand distance vector (AODV) protocol based on the computed connectivity information; network longevity, packet delay and packet drop rate are obtained through triggering events in WSNet simulator according to a user defined sensing task and the provided topology. It is the first time that network simulator is involved in a planning algorithm to tackle the difficulty on modelling those metrics and provide practical evaluations.

- Efficient and multi-objective optimization. A multi-objective optimization algorithm is developed for WSN to optimize the cost, coverage, lifetime, packet delay and packet drop rate. The individual length is changeable so that the cost can be optimized, meanwhile crossovers and mutations are designed to eliminate invalid modifications and improve the computation efficiency. NSGA-II ranking method, which is proved with high efficiency, is employed by this work.

- Solid results for both indoor and outdoor WSN planning. Real deployments are realized for both indoor and outdoor environments based on the provided planning solutions. The measured results coincident well with the estimated results. The proposed planning algorithm is adaptable according to the WSN designer's 
desirability and configuration, and it supports a flexible planning to small and large scale, indoor and outdoor 3D deployments.

The remainder of this paper is organized as follows: in Sect. 2 the modelling of important metrics that impact the performance of WSNs is introduced. The proposed multi-objective optimization method is presented in Sect. 3, experiments and comparisons for simulations and real deployments are realized and analyzed in Sects. 4 and 5 respectively. Section 6 draws the conclusions and describes future works.

\section{Modelling of important metrics}

\subsection{Preliminaries and assumptions}

The important symbols that are used in this work are summarized in Table 1. The proposed method employs a ray-tracing method described in the authors' previous work (He et al. 2012) to emulate the propagation paths of both radio and sensing signals. In the real world propagation, multi-path phenomena occur on the radio signal transmission: when a radio signal encounters obstacles, reflections and diffractions happen. Thus RSS at the receiver (RX) is computed by accumulating the arrived waves from all directions. In the implemented ray-tracing method, the obstacles of the deployment field is polar swept (see Fig. 3) by bending the direction of the line whenever intersection occurs. A $3 \mathrm{D}$ line is rotated clock wisely and bottom up centering at the transmitter (TX) to discover for each direction the first intersected point and its corresponding plane. The rule of reflection is then applied and intersected planes are recorded whenever the direction changes at halting points. This procedure is repeated for each candidate direction, and each $3 \mathrm{D}$ line terminates shooting after a maximum number of intersections is reached or when the boundary is touched. Therefore, by sweeping the entire scene, all the possible orders of reflection planes and diffraction cones are discovered. The order of reflected plane is stored as a matrix Ref_plane with dimension of $N * d e p t h$, where $N$ is number of possible reflection paths, depth is the maximum depth of reflections predefined in the ray tracing engine. When visible

Table 1 Important symbols

\begin{tabular}{ll}
\hline Symbol & Meaning \\
\hline$S_{i}$ & Sensor node with $I D=i$ \\
$N_{i}$ & Node device with $I D=i$ and $S \in N$ \\
$A_{S}$ & Sensing/monitoring area \\
$\Phi_{S_{i}}$ & A set of points that are the covered points of $S_{i}$ \\
$C$ & Coverage \\
cost & Cost \\
$L$ & Lifetime \\
$P_{l}$ & Packet latency \\
$P_{d}$ & Packet drop rate \\
$D_{C, \cos t, L, P_{l}, P_{d}}$ & desirability formulation of $C, \operatorname{cost}, L, P_{l}, P_{d}$ \\
\hline
\end{tabular}




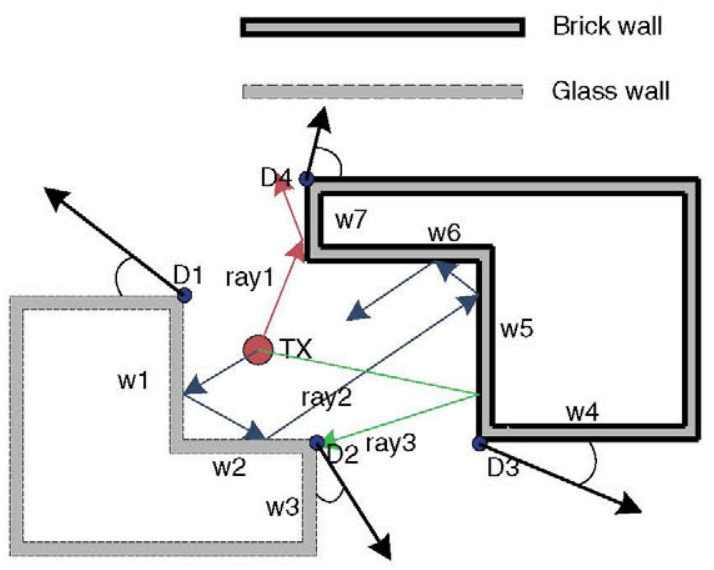

plane exists and the length is less than depth, the ID of intersected primitive plane is recorded in a order along the signal path, such as $w 1 \rightarrow w 2 \rightarrow w 5 \rightarrow w 6$. When the number of planes in a path is less than depth, NULL is assigned to the remaining elements:

$$
\text { Ref_plane }=\left[\begin{array}{cccc}
w 7 & \text { NULL } & \text { NULL } & \text { NULL } \\
w 1 & w 2 & w 5 & w 6 \\
w 5 & w 3 & \text { NULL } & \text { NULL } \\
\vdots & \vdots & \vdots & \vdots
\end{array}\right]
$$

Diffraction happens at the convex edges, the diffraction points are extracted based on the resolution of $\mathrm{z}$ direction and Dif_cone is a structure used to store all the information of a diffraction cone.

At the end, according UTD theory, the total received electric field $E_{t o t}$ at $P$ is the combination of direct path $E_{d i r}$, reflected path $E_{r e f}$ and diffracted path $E_{d i f}$.

$$
E_{\text {tot }}=E_{\text {dir }}+E_{\text {ref }}+E_{\text {dif }}
$$

Unlike the radio propagation, the sensing signal usually only has direct path, which halts at the intersected points with the surfaces of obstacles. Thus a covered point of a sensor node $S_{i}$ is defined as follows:

Definition 1 Covered point: A point $m$ is said to be covered by $S_{i}$, if and only if it is within the sensing range of $S_{i}$ and is not obstructed by any obstacle. $\Phi_{S_{i}}$ represents a set of all the points that are the covered points of $S_{i}$.

$$
\Phi_{S_{i}}=\left\{m \mid m \in \operatorname{sphere}\left(S_{i}, r_{S_{i}}\right) \wedge ! O\left(\overrightarrow{m S}_{i}\right)\right\}
$$

As indicated in Fig. 4, sphere $\left(S_{i}, r_{S_{i}}\right)$ is the sphere with radius $r_{S_{i}}$ centered at node $S_{i}$ and indicates the ideal sensing area of $S_{i} . O\left(\overrightarrow{m S_{i}}\right)$ indicates whether the sensing path from $m$ to $S_{i}$ is obstructed. 
Fig. 4 The searching of covered point

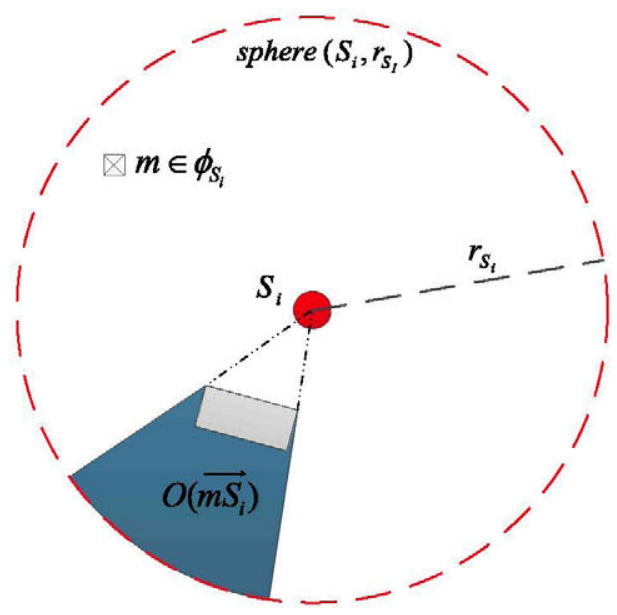

Routing scheme: There have been many algorithms proposed for routing data in sensor networks, which consider the characteristics of sensor nodes together with the application requirements. Nowadays, an overwhelming number of commercial sensor devices support the distance vector based routing protocol such as the AODV Routing (Perkins et al. 2003) and link-state based routing protocol dynamic source routing (Johnson et al. 2007). However, the routing results of minimum-weight/minimum-hop based routing protocols are very similar and can be computed based on the shortestpath searching by Dijkstra's algorithm, in which data are collected and forwarded to BS via the path with the best distance metric. In this work, the distance metric is modelled by the qualities of established links according to the ray-tracing results, which not only considers the distance between transmitter and receiver but also indicates the impact of surrounding environment.

\subsection{The cost of WSN}

One of the design goal, from economic point of view, is to reduce the cost while fulfilling requirements of an application. Many companies and research organizations arise in the recent decade to design and manufacture sensor nodes, which provide various options on the budget. Besides, the installation of sensor nodes requires extra human efforts. For instance, the cost of mounting sensors on the ceiling and walls is different from mounting them within a human-active space; even attaching sensor nodes to different heights can vary the costs. As a result, the Cost of WSN is categorized into hardware cost and deployment cost in this work.

\subsubsection{Hardware cost}

MICA2 was one of the most successful families of Berkeley motes (Beutel et al. 2003), whose platform is equipped with Atmel ATmega128L and a CC1000 transceiver. The newer generations MICAz and Telos (Polastre et al. 2005) support IEEE 802.15.4, 
Table 2 Features of various platform

\begin{tabular}{llllll}
\hline Platform & CPU & Communication & External memory & Power supply & Price \\
\hline Mica2 & ATMega128L & CC1000 & $512 \mathrm{kB}$ Flash & $2 \times$ AA & $\$ 150$ \\
Telos & MSP430F149 & CC2420 & $512 \mathrm{kB} \mathrm{Flash}$ & $2 \times$ AA & $\$ 110$ \\
IMote2 & Intel PXA271 & CC2420 & $32 \mathrm{M} \mathrm{Flash}$ & $2 \times \mathrm{AA}$ & $\$ 299$ \\
BTnode rev3 & ATMega128L & Bluetooth CC1000 & $128 \mathrm{~KB}$ Flash & $2 \times$ AA & $\$ 215$ \\
Waspmote & ATmega1281 & 8 radio modules & $128 \mathrm{~KB}$ Flash & $2 \times$ AA & $\$ 130 \sim \$ 300$ \\
\hline
\end{tabular}

Fig. 5 The architecture of 'Cookie' node

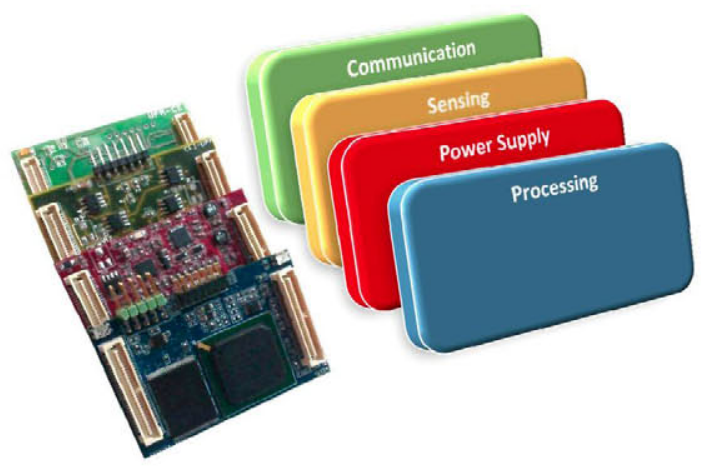

and the unit cost is about $\$ 100 \sim \$ 200$. Intel has designed its own iMote (Kling 2003) with a powerful ARM7TDMI core complemented by a large main memory and non-volatile storage area; on the radio side, Bluetooth has been chosen, thus the price of an iMote is about $\$ 299$, which is very high. Waspmote (2013) works with different communication protocols (ZigBee, Bluetooth and GPRS) and frequencies $(2.4 \mathrm{GHz}$, $868 \mathrm{MHz}, 900 \mathrm{MHz}$ ). It is compatible with more than 50 sensors and the price varies with different hardware configurations. For example, sensors with ZigBee module cost the least compared with that attached with Wi-Fi and Bluetooth module, while the one with $3 \mathrm{G}+\mathrm{GPS}$ costs around $\$ 300$ and is the most expensive configuration. Table 2 summarizes the hardware features of some famous sensor productions and their prices, which significantly depend on the hardware configurations and designs.

Beyond the on-shelf sensor products, there are enormous research organizations dedicating to develop and prototype their own sensor platforms. Typically, those prototypes are based on add-on/modular hardware design, which is not highly optimized in terms of power consumption or size and price. Such as the Tyndall's mote family (Tyndall 2013), and Cookies developed by Portilla et al. (2006) at Centro de Electronica Industrial of Universidad Politecnica de Madrid (CEI-UPM), have a modular architecture of four layers. As depicted in Fig. 5, each layer in "Cookie" fulfills a specific functionality in the node, and the layers are changeable for different applications. Moreover, it is possible to have a heterogeneous network with nodes composed of different layers. In the current motes, $2.4 \mathrm{GHz}$ ZigBee communication protocol is used and AODV routing protocol is embedded. 
Due to the heterogeneity property and various topologies of WSNs, motes with different functions should be considered to obtain a optimum cost solution rather than simply using a uniform type of all-function sensor mote for the whole network. Based on the above survey, motes are classified into three types in this work:

- Sensor node (SN): equipped with sensors to monitor the surrounding environment. In this work, each sensor node is static and has wireless communication and routing ability.

- Relay node (RN): has the ability of communication and routing. RN is usually needed to fill radio communication hole or to balance traffic load.

- Base station (BS): is in charge of aggregating data and is directly connected with the central server. A WSN has one BS and its location is predetermined by users.

Since the number and location of BS is assumed to be fixed in this work, the cost of BS is also fixed and is not included in the model of hardware cost. SN contains extra sensor module besides the communication and routing module, therefore a SN costs more than a RN. The hardware cost model $\operatorname{cost}_{h} w$ is expressed by accumulating the price of each node deployed in the area $A$ :

$$
\operatorname{cost}_{h w}=\sum_{i=1}^{M}\left(P\left(N_{i} . t y p e\right)\right)
$$

where $P\left(N_{i} . t y p e\right)$ indicates the relative price that is dependent on the type of node $N_{i}$, for instance $P(R N)=1, P(S N)=3$.

The available budget is considered as the maximum cost $\operatorname{cost}_{\text {max }_{h w}}$. The normalization of $\operatorname{cost}_{h} w$ provides a desirability component of the design goal to minimize the hardware cost, and $D_{\text {cost }}$ is used in this work to represent the desirability on hardware cost.

$$
D_{\text {cost }}=\frac{\cos _{\text {max }_{h w}}-\operatorname{cost}_{h w}}{\operatorname{cost}_{\max }}
$$

\subsubsection{Deployment cost}

The investigation on the lessons learnt by other researchers indicates that mounting problem occurs in all the deployments. Mafuta et al. (2012) deployed WSN for precision agriculture, and they observed that multi-path fading, which was exacerbated by the movement of leaves of the maize plants played a very crucial role on RSSI. Langendoen et al. (2006) tried to deploy 100+ sensor nodes to monitor the potato crops for precision agriculture. To avoid obstruction when the potato crop is flowering and leaves cover the (ground-based) antennas, the nodes were installed on poles with a height of $75 \mathrm{~cm}$. Besides they included a safety margin to ensure that the nodes could not be hit by farming equipment attached to a tractor. Lau et al. (2006) also learned very interesting lessons, they developed a WSN to monitor the indoor environment. However, they found in two occasions that the sensor nodes are taken from where they were. As a result, they decided to place the sensor nodes not for the best coverage but for the best security. Hence those sensor nodes are eventually either hidden from field of vision or placed high up on the wall. 
Fig. 6 Deployment cost configuration in vertical view

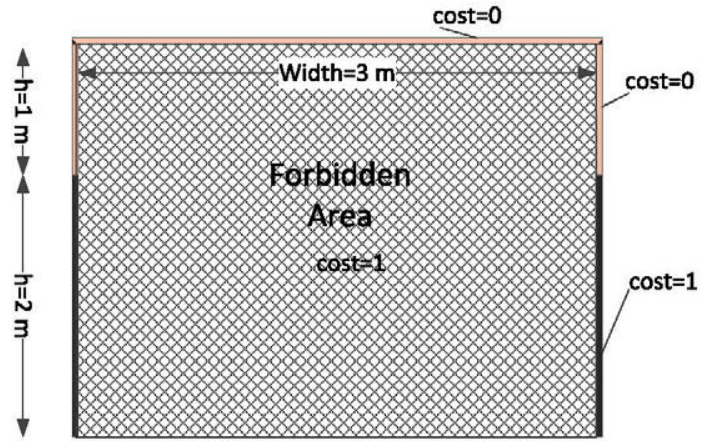

There might exist non-deployable area where nodes are not allowed to be placed, those area should be pre-defined and the corresponding deployment cost can be assigned with the maximum value. In this work, deployment cost cost $_{d}$ is modeled as boolean value and the forbidden area is assigned by users through a GUI interface as shown in Fig. 6, cost $_{d}=1$ if the area is not accessible, otherwise $\operatorname{cost}_{d}=0$.

\subsection{Coverage}

Sensing coverage is one of the key issues that should be considered when deploying a WSN, as it corresponds to the quality of service that can be provided by a WSN. The coverage concept can be defined and categorized based on the node density level. If only parts of the area are covered by the sensor nodes, the coverage is sparse; if the area is completely (or almost completely) covered by sensors, the coverage is dense; otherwise, if the same detected location is covered by multiple sensors, the coverage is called redundant. The density of coverage is normally determined by user requirements, which may vary across different applications. An adequate coverage is a key to a robust WSN application, and it may also be exploited to extend the network lifetime by switching redundant nodes to sleep modes to reduce power consumption. In this work, K-coverage problem is investigated and is concerned as the potential problem to be tackled by planning algorithm.

Definition 2 Target K-coverage Requirement: At any given moment, any target point $m \in A_{s}$ is the covered point of at least $k$ different SNs $(k=1 \cdots M)$.

Assuming that the number of target points within $A_{s}$ is $N_{m}$, the desirability of $\mathrm{K}$-coverage requirement $C$ is expressed as:

$$
D_{C}=\frac{\sum_{j=1}^{N_{m}} \frac{\sum_{i=1}^{M}\left|m_{j} \in \Phi_{S_{i}}\right|}{k}}{N_{m}}
$$

where $|\cdot|$ indicates whether the insider expression is true. 


\subsection{Connectivity}

WSNs are represented by a graph $G=(V, E)$ where $V$ is the set of nodes and $E \subseteq V^{2}$ is the set of edges: $\left(N_{u}, N_{v}\right) \in E$ means that $N_{u}$ and $N_{v}$ are neighbors. The neighborhood set $\mathfrak{N}(u)$ of $N_{u}$ is expressed by

$$
\mathfrak{N}(u)=\left\{N_{v} \mid\left(N_{u}, N_{v}\right) \in E \vee\left(N_{v}, N_{u}\right) \in E\right\}
$$

wireless links are determined according to RSS calculated by the accurate ray-tracing method, thus edges are defined as :

$$
E=\left\{\left(N_{u}, N_{v}\right) \in V^{2} \mid u \neq v \wedge R S S(u v) \geq R X_{s}\right\}
$$

where $R S S(u v)$ is the RSS from $N_{u}$ to $N_{v}$, and $R X_{s}$ is the sensitivity of antenna at the receiver.

As can be seen from the edge definition, communication links can be established if the RSS is above the sensitivity of antenna. A WSN is said to be connected, if any two nodes belong to a WSN are linked together by edge(s) via single hop or multiple hops. Connectivity is intermittent if the network is occasionally partitioned. If nodes are isolated most of the time and enter the communication range of other nodes occasionally, the communication is said sporadic (Romer and Mattern 2004). Note that despite the existence of partitions, messages may be transported across partitions by mobile nodes, which is not the case in this work where only static network is considered. Connectivity mainly influences the design of communication protocols and methods of data gathering. Generally speaking, the concept can be categorized into two directions:

Definition 3 Connected K-Coverage Problem: Given a sensor network consisting of $\mathrm{n}$ sensors and an interest region, the network should satisfy the following two conditions at any moment:

\section{Satisfy the conditions of the K-Coverage requirement}

2. The communication graph $G$ is connected

Definition 4 K-connected Problem: A graph $G$ is said to be k-connected if for each pair of vertices there exist at least $\mathrm{k}$ mutually independent paths of edges connecting them. In other words, the graph $G$ is still connected even after removal of any $k-1$ vertices from $G$.

In this work, connectivity of WSN is constructed to tackle the Connected K-Coverage Problem with K-connected network topology by using the previous mentioned sensing model, practical multi-path radio propagation model on heterogeneous WSNs. 


\subsubsection{Lifetime}

As most of the sensor nodes are powered by batteries, they will exhaust energy after a certain time once deployed in the environment. Therefore the WSN will be disconnected and no longer satisfy the sensing requirement. The lifetime of sensor network is a very important metric and WSN designers have done many efforts to prolong it. The methods include developing proper MAC periods, optimizing the topology to reduce bottleneck nodes, developing back up plans and routing algorithms. The network lifetime in this work is defined as the time that the first node exhausts its energy. The desirability of this metric is expressed by a ratio between the actual lifetime $(L)$ and the maximum expected lifetime $\left(L_{\max }\right.$ ) by the WSN designer. The expression of $D_{L}$ is:

$$
D_{L}=\frac{L}{L_{\max }}
$$

\subsubsection{Packet latency}

Packet latency is defined as an average end-to-end delay from the source to the destination (BS). There are many factors that affect packet latency and the most important factors are: the usage of channel, the hops between source and destination and the scheduling of nodes along the routing path. The desirability of packet latency is expressed as:

$$
D_{P_{l}}=1-\frac{\sum_{i}^{M_{p}} P_{l}(i)}{M_{p}}
$$

where $M_{p}$ is the total number of data packets generated by all the sensors and $P_{l}(i)$ is the latency of packet $i$.

\subsubsection{Packet drop rate}

Packet drop can be caused by signal degradation over the network medium due to multi-path fading, channel congestion, corrupted packets rejected in-transit, faulty networking hardware, faulty network drivers or invalid routes. The packet drop rate is a ratio between the number of dropped packet $\left(P_{d}\right)$ and the number of generated data packets, and the desirability over this metric is:

$$
D_{P_{d}}=1-\frac{P_{d}}{M_{p}}
$$

\subsubsection{Proposed strategy by using WSNet simulator to model L, $P_{l}$ and $P_{d}$}

Because $L, P_{l}$ and $P_{d}$ can be affected by network topology, real-time communication and packet load, it is difficult to precisely model them through simple formulas. That is the reason why protocol designers usually estimate such performance through network 


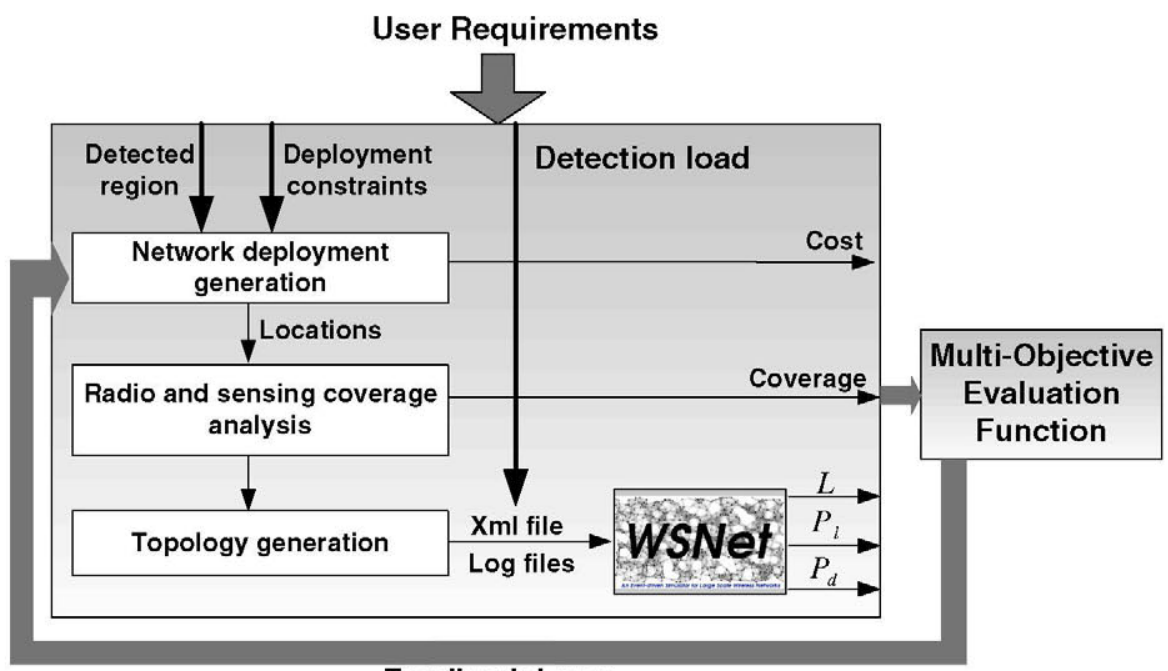

Feedback Loop

Fig. 7 The strategy of planning method

simulators, where WSN can be simulated with a determined or random topology with nodes being scheduled and data load being assigned based on user specifications. Inspired by those works, WSNet ${ }^{1}$ simulator is integrated in this work to observe the three complex metrics through practical network simulation. WSNet simulator allows researchers to analyze WSN performance based on network configuration, which can be read from the files that indicate topology, routing and connectivity information. Users can either use the embedded provided layer modules or extend the operation by developing customized modules that will be used in the real application. The network protocol can be the 802.15.4 standard for physical layer and MAC layer, or other desired protocols. The energy model should be programmed previously to imply how energy is consumed for transmitting/receiving data packets and for different status such as wake, sleep and idle.

As a result, the strategy of the modelling method by WSNet simulator can be described by the lower part of the flowchart in Fig. 7. When a candidate topology is generated, the node location, routing and connectivity files are created in a shared folder between the planning algorithm engine and the WSNet simulator. A 'xml' script is created to configure the WSN properties for the simulation in WSNet, including the network size, region scale, network protocols, energy consumption models and directory of the generated output files. After WSNet finishing the simulation, it returns the values of network lifetime, packet latency and packet drop rate to the planning algorithm engine, for analyzing desirability values by the aforementioned formulas $(10,11$ and 12).

1 http://wsnet.gforge.inria.fr/ 


\section{The proposed multi-objective optimization methodology}

Once provided application requirements and deployment environment model, the problem of planning a WSN is formulated as: Determine the topology of the network to maximize the five desirability values calculated by $(6 \sim 12)$. This is a multi-objective optimization problem, which is proven to be NP-hard (Efrat et al. 2004; Cheng et al. 2008; Poduri et al. 2006). Multi-objective optimization genetic algorithms are proposed to effectively and efficiently solve the NP-hard problems, among which the Nondominated Sorting Genetic Algorithm II (NSGA-II) by Deb et al. (2002) is an ideal approach that features with elitism selection, high computation efficiency $O\left(M N^{2}\right)$ (where $M$ is the number of objectives and $N$ is the population size) and does not need to specify the sharing parameter. As a result, the multi-objective optimization method in the present work is based on NSGA-II. It concerns the constraints and rules on formulating the genes as well as the mutations and crossovers, to cater to the features of WSN, and therefore the results are achieved efficiently and effectively. NSGA-II is realized through three steps, the first step is crowded tournament selection. A solution $x_{i}$ wins a tournament with another solution $x_{j}$ if any of the following conditions are true:

- If solution $x_{i}$ has a better rank, that is, $r_{i}<r_{j}$.

- If they have the same rank but solution $x_{i}$ has a better crowding distance than solution $x_{j}$, that is, $r_{i}=r_{j}$ and $d_{i}>d_{j}$.

The second step is crowding distance, which is to get an estimate of the density of solutions surrounding a particular solution. The third step is the crowding distance assignment. Assuming that $F$ is a set of solutions in a front, its length $l=|F|$, let the distance of each solution $d_{i}=0$ from the beginning, where $i=1,2, \ldots, l$. Then, for every objective function $m=1,2, \ldots, M$, sort the set $f_{m}$ in descend order, or find the sorted indices vector: $I^{m}=\operatorname{sort}\left(f_{m}\right)$. For $m=1,2, \ldots, M$, assign a large distance to boundary solutions, i.e. set them to $\infty$, and for all the other solutions(from 2 to $l-1$ ), the distance is assign as follows:

$$
d_{l_{j}^{m}}=d_{l_{j}^{m}}+\frac{f_{m}^{I_{j+1}^{m}}-f_{m}^{I_{j+1}^{m}}}{f_{m}^{\max }-f_{m}^{\min }}
$$

Figure 7 shows an overview of the proposed planning method. Network deployment is generated based on the deployment constraints and user requirements on the detected regions and forbidden regions, the value of cost is obtained at this step. There are three ways to create the deployment:

1. User can determine the locations and properties of nodes manually via the GUI interface;

2. Nodes can be randomly generated based on the constraints;

3. Crossover and mutation modify the node properties during the evolutionary strategy.

Thereafter, radio propagation and sensing signals are computed for each node by using the ray-tracing method so that the connectivity and sensing coverage are 
obtained, and the topology of the WSN is constructed according to the routing protocol pre-defined by WSN designer. As discussed in Sect. 2, the 'xml' file and log files are generated to trigger WSNet simulator so that lifetime, packet latency and packet drop rate are analysed after WSNet finishing simulation. With all those metrics provided, the multi-objective evaluation method computes the objectives (desirability) and selects those candidates with best performance based on NSGA-II, and the selected population are fed back to the network deployment generation function to create new populations, thus the objectives are gradually progressed. At the end, the algorithm can provide multiple elitist solutions to WSN designers.

Generally speaking, in a genetic algorithm, a candidate solution can also be called individual, creature, or phenotype. Each candidate solution has a set of properties (its chromosomes or genotypes), which can be mutated and altered. Traditionally, individuals are represented in a vector of binary value, but other representations are also possible. The evolution usually starts from a population of randomly generated individuals and in each generation, the fitness value of every individual in the population is evaluated. The individuals with better performance are selected from the current population, and each individual's genome is modified (recombined and possibly randomly mutated) to form a new generation to be evaluated in the next iteration. Commonly, the algorithm terminates when either a maximum number of generations has been produced, or a satisfactory fitness level has been reached.

In this work, an individual is expressed as $D V$ represented by (14), where $N_{i}$ is the node with $I D=i$ and it is a "chromosome" of $D V . M$ is the number of nodes, which also indicates the size of WSN. Each "chromosome" has properties including 3D location of the node, type of node, transmission power $P_{t x}$, radio sensitivity $R X_{s}$ and sensing range $R_{\text {sense }}$. It is assumed in this work that $M, N_{i}$.location, $N_{i} . t y p e$ can be modified, as a result this method is based on changeable length, which will bring difficulties to produce crossover and mutation. Besides, the location and type must be modified according to a certain rules to construct a valid WSN. The details are introduced and problems are tackled later in this section.

$$
D V=\left[N_{1}, N_{2}, \cdots N_{M}\right], N_{i}=\left\{\begin{array}{l}
\text { location }: x, y, z \\
\text { type }: B S, S N, R N \\
P_{t x}, R X_{S} \\
R_{\text {sense }}
\end{array}\right\}
$$

The algorithm defines two types of population: Parent and Children, for storing the parents and children respectively. The format of population is defined by $N_{p}$ individuals attached with their corresponding desirability values:

$$
\text { population }=\left[\begin{array}{cc}
D V_{1}, & D_{\text {cost }, c, L, P_{l}, P_{d}}^{1} \\
D V_{2}, & D_{\text {cost }, c, L, P_{l}, P_{d}}^{2} \\
\cdots & \\
D V_{N_{p}}, & D_{\text {cost }, c, L, P_{l}, P_{d}}^{N_{p}}
\end{array}\right]
$$




\subsection{Initialization of individuals}

Initial population of candidates are traditionally generated in a random way, and some of them may occasionally satisfy the constraints and requirements, not to mention optimizing the performance at the same time. When the scale of region or the size of WSN becomes large, there will be less chance that an initial candidate has a valid WSN topology. To efficiently tackle this issue, a high "quality" initial seed is generated to guarantee the basic requirements on connectivity and coverage. The LowCost heuristic proposed by Kouakou et al. (2010) is modified to add a valid individual at initial phase:

At the beginning, the Coverage is computed for each deployable point $m \in A_{t}$ and the heuristic selects $m_{i}$ that with the maximum Coverage as the best location and a sensor node $S_{u}$ is then placed on $m_{i}$. The coverage level of the monitoring points newly covered by $S_{u}$ is updated, and those points with a sufficient coverage level are removed from the set of sensing area $A_{s}$. This procedure is repeated until all the monitoring points are k-covered.

Afterwards, LowCost focuses on the connectivity problem. Let $N_{u}$ be the node of unconnected nodes $\mathfrak{U}$. The algorithm selects a node $N_{c}$ in the connected sensor nodes $\mathfrak{C}$ that is the closest to $N_{u}$ and computes the new virtual position $m^{\prime}$ of $N_{u}$ by moving it towards $N_{c}$ as long as the set of monitoring points initially covered by $N_{u}$ remains unchanged. If $N_{u}$ is still unconnected after changing its position, extra relay nodes are put on the line between $N_{u}$ and $N_{c}$ so that $N_{u}$ and $N_{c}$ are connected.

The resulted initial seed is expected to be better than a randomly generated seed, which do not guarantee the coverage and connectivity. However, as discussed in Sect. 1 , the result does not solve the optimization between connectivity and cost. Besides the individual generated by LowCost heuristic, the rest individuals are generated based on the constraints on location with various length (size of WSN), thus the initial population of parents (Parent) are obtained and evaluated.

\subsection{Crossover and mutation}

At each generation, the parents are recombined (crossover) and mutated with different probability. The demonstrations of crossover and mutation with variable individual lengths are shown in Figs. 8 and 9 respectively.

\subsubsection{Crossover}

Two candidates (Parent $(i)$ and $P \operatorname{arent}(j))$ are randomly selected from the population of parents and crossover occurs between them with a chance of $P_{c o}$. In this work the crossover procedure applies two-point strategy, thus crop length and offset index are needed to define the crossover segments. Since the length of both parents might be different, the crop length $L_{c r o p}$ is limited by the shorter length of the two parents: $L_{\text {crop }}<\min \left(M_{i}, M_{j}\right)$, and is randomly generated between 0 and $\min \left(M_{i}, M_{j}\right)-1$. The offset value $I_{o f f}$ is also randomly generated for each individual of the selected crossover pair, with constraint: $I_{o f f}(i)+L_{\text {crop }} \leq M(i)$ and $I_{o f f}(j)+L_{\text {crop }} \leq M(j)$. Afterwards, the two generated children $\left(A^{\prime}\right.$ and $\left.B^{\prime}\right)$ are stored into the population of 


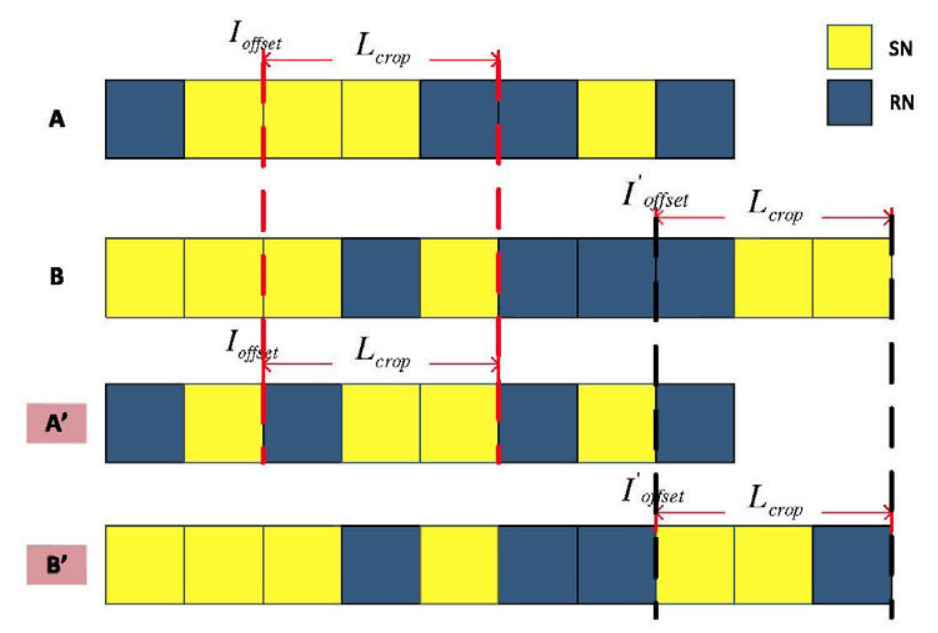

Fig. 8 Crossover with different lengths

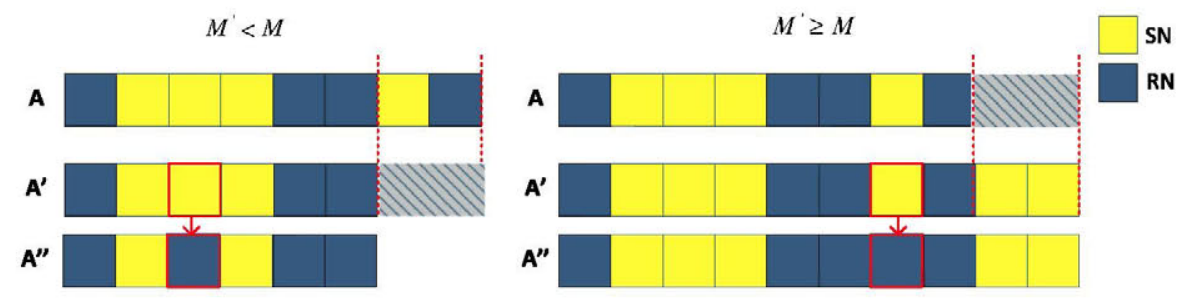

Fig. 9 Mutation with changeable length

Children. If crossover does not happen on the current couple, the two parents are stored into Children directly for further modification. Similar procedure repeats on other possible pairs of Parent until Children is filled by individuals.

\subsubsection{Mutation}

Mutation occurs on the individuals of Children. The size of Children(i) is $M_{i}$, which mutates with a probability of $P_{m u}$. Note that the change on $M_{i}$ will to some extent increase the risk of constructing an un-connected topology. The new length $M_{i}^{\prime}$ is obtained by randomly selecting a value within the range limited by $\left[T_{\min }, T_{\max }\right]$, where $T_{\min }=\max \left(M_{i}-T, 1\right)$, and $T_{\max }=\min \left(M_{i}+T, M_{\max }\right) . T$ is a small integer value, which can be determined arbitrarily by WSN designers. The purpose of constraining the variation range with $T$ is to reduce the chance of constructing an un-connected WSN and to gradually improve the performance of populations. $T$ is equal to 2 in this work. $M_{\max }$ is the maximum number of nodes that allowed to be used and is determined by users. If $M_{i}^{\prime}$ is less than $M_{i}, M_{i}^{\prime}$ nodes are randomly picked from Children $(i)$; otherwise, if $M_{i}^{\prime}>M_{i}, M_{i}^{\prime}-M_{i}$ random nodes are added to Children $(i)$. 
Thereafter mutation happens on each node of Children $(i)$ with probability of $P_{m u}$, on the 3D location and the type of node. The movement of $N_{j} \in$ Children (i) is limited within the sphere of radius $d_{\max }$ centered at $N_{j}$.location and the type can be selected randomly between $\mathrm{SN}$ and RN.

\subsection{Evaluation based on desirability models and constraints}

Routes are searched from each node to the BS by using the Dijkstra's method. Several further steps are prosecuted to make the algorithm converge faster: a $\mathrm{SN}$ is changed to $\mathrm{RN}$ when it does not cover any point $m \in A_{s}$; a RN is deleted if it does not act as a router for other nodes; if two nodes of the same type are located too close to each other, one of them is moving apart in a similar way as the mutation on location.

The desirability values of all the 5 metrics are computed and then attached to each corresponding individual. Parent and Children are mixed so that all the individuals from both populations are ranked based on nondominated sorting by NSGA-II. As a WSN must focus on fulfilling the sensing tasks, the desirability of coverage $D_{C}$ is considered as the only constraint among the five objectives. By doing so, if $D_{c}^{i}>D_{c}^{j}$, the rank of individual $i$ is always higher than individual $j$ no matter how the other metrics are; otherwise, the ranking is based on all the desirability values equivalently, the greater a desirability is, the better the corresponding metric will be. At the end of each generation, $N_{p}$ best individuals are selected and formulate new Parent for the next generation.

The evolutionary procedure repeats until the maximum generation is reached. The proposed method is able to provide multiple WSN deployment solutions with optimized performance from different aspects. As a result, it gives designers flexibility to observe different optimized deployments and assist them making deployment decisions accordingly.

\section{Simulation results and analysis}

The performance of the planning algorithm is evaluated through observing the fitness value and time efficiency compared with other heuristics. The performance comparisons are realized with three comparable state-of-the-art algorithms, which have 3D computation ability. All the algorithms including the proposed one are programmed in $\mathrm{C}++$ and they are run on a PC with Intel Core i5-760 2.8 GHz CPU so that the results are fairly compared.

\subsection{The impacts of maximum number of generation, population size and evolutionary possibilities}

As the proposed algorithm is based on evolutionary strategy, the larger the number of generations, the more outstanding the population will evolve. Therefore, this work first evaluates how the maximum number of generations $N U M_{\max G e n}$ impacts on WSN performance. The application requirements are shown in Fig. 10: The 3D map 


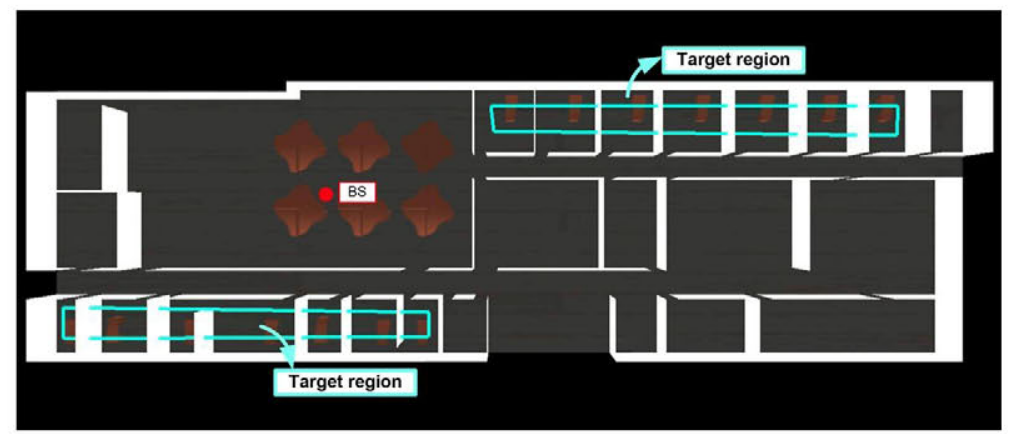

Fig. 10 Scenario CEI-UPM

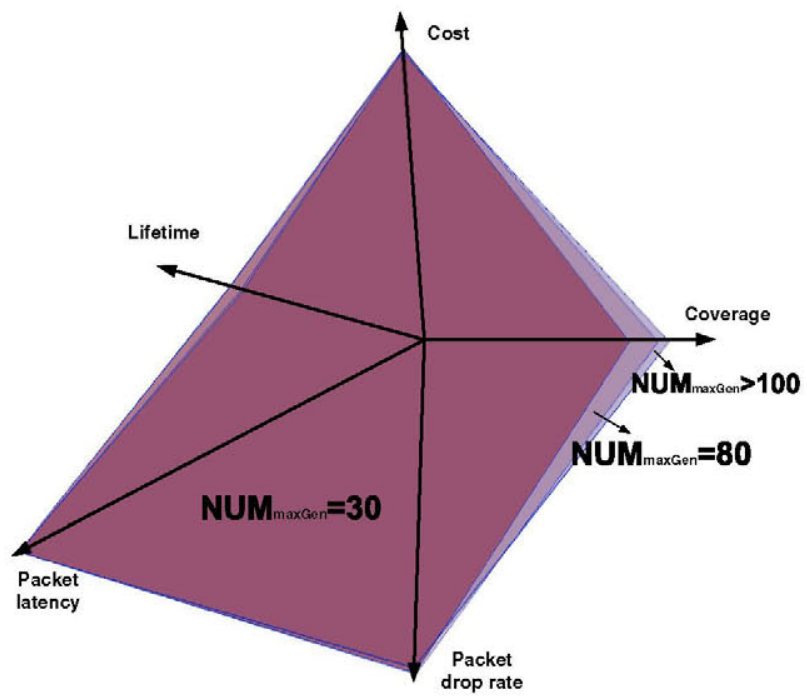

Fig. 11 Desirability values vary with $N U M_{\max G e n}$

is the floor plan of CEI-UPM with a scale of $57 \times 16 \times 3 \mathrm{~m}^{3}$ and a resolution of $1 \mathrm{~m}$. The red point indicates the location of BS $(17.52,10.02,1.5 \mathrm{~m})$ and blue rectangles are the sensing areas $A_{s}$, which contain 70 points to be covered. In this study, the size of population is $N_{p}=8$ for both Parent and Children. Crossover and mutation possibilities are $P_{c o}=0.1$ and $P_{m u}=0.2$ respectively. The data period for each sensor node is $1 \mathrm{~s}$ and the simulation lasts $2,400 \mathrm{~s}$ in WSNet simulator.

The maximum value of generation $N U M_{\max G e n}$ increases from 10 to 150 with a step of 10 , hence there are 15 different $N U M_{\max G e n} \mathrm{~s}$. The algorithm runs 5 times for each $N U M_{\max G e n}$, and as a result $8 \times 5 \times 15$ optimized solutions are obtained after the simulation.

The results are grouped for each $N U M_{\text {maxGen }}$ and the pareto front is shown in Fig. 11, the mean value of each group data are calculated for each desirability metric, the five metrics construct a plot with five axes. The area constructed from each group indicates that as $N U M_{\max G e n}$ increases and the area grows larger, and the overall 


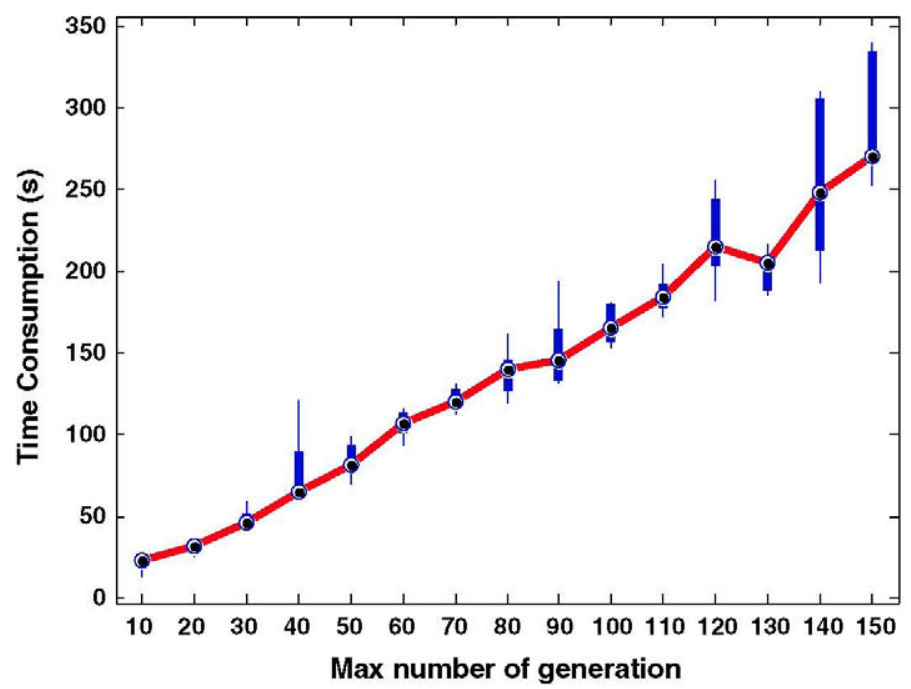

Fig. 12 Time consumption varies with $N U M_{\max G e n}$

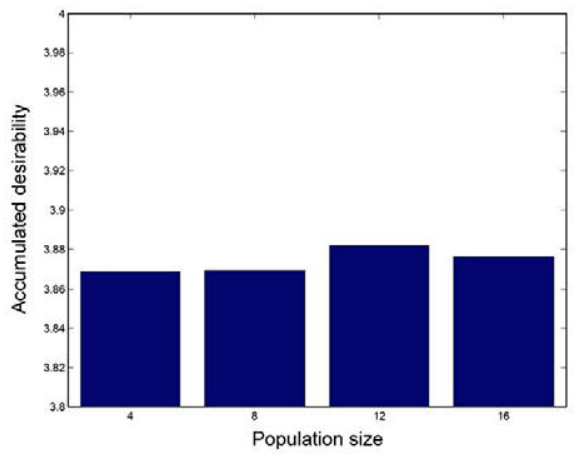

(a)

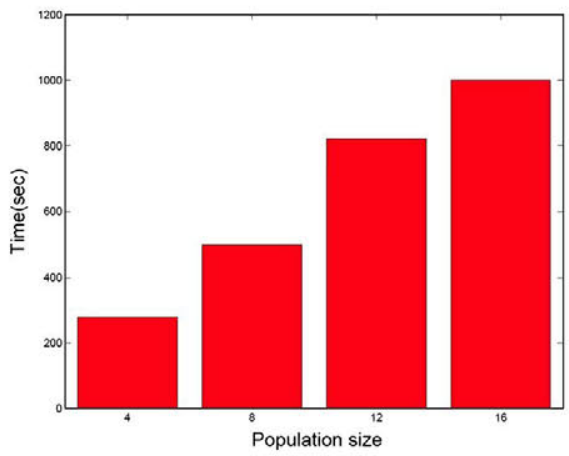

(b)

Fig. 13 Impact of population size. a Population size affects the optimization performance. b Population size affects the computation time

performance becomes more stable when $N U M_{\max G e n} \geq 100$. Figure 12 indicates that although time consumption fluctuates at the same $N U M_{\max G e n}$, consumed time increases approximately linearly with $N U M_{\max G e n}$. Therefore, a proper trade-off decision should be made between the performance and efficiency of computation, and $N U M_{\max G e n}=100$ can be selected for this configuration.

The population size effects the desirability performance and computation efficiency as indicated in Fig. 13. The $N U M_{\max G e n}=100$ in this test, the larger the population size is, the higher the desirability will be, however, the improvement might fluctuate and grows slowly, whereas the computation time is increased significantly with the size, as a result, there should be a trade off between the optimization performance and computation time. The mutation and crossover possibilities are studied by setting $N U M_{\max G e n}=100, N_{p}=8$, the results in Figs. 14 and 15 indicate that their 


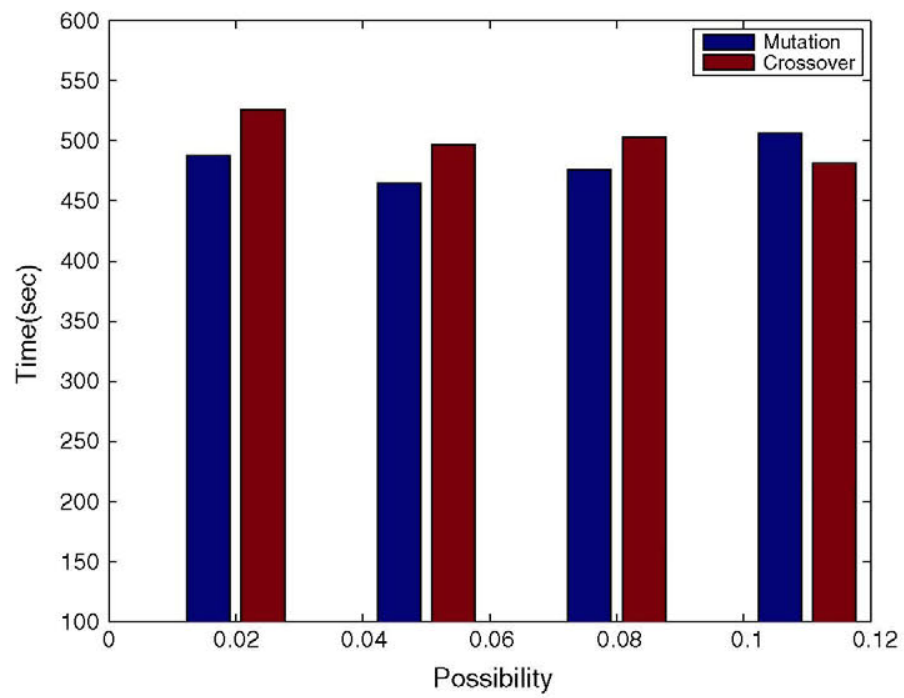

Fig. 14 The impact of mutation and crossover possibilities over time

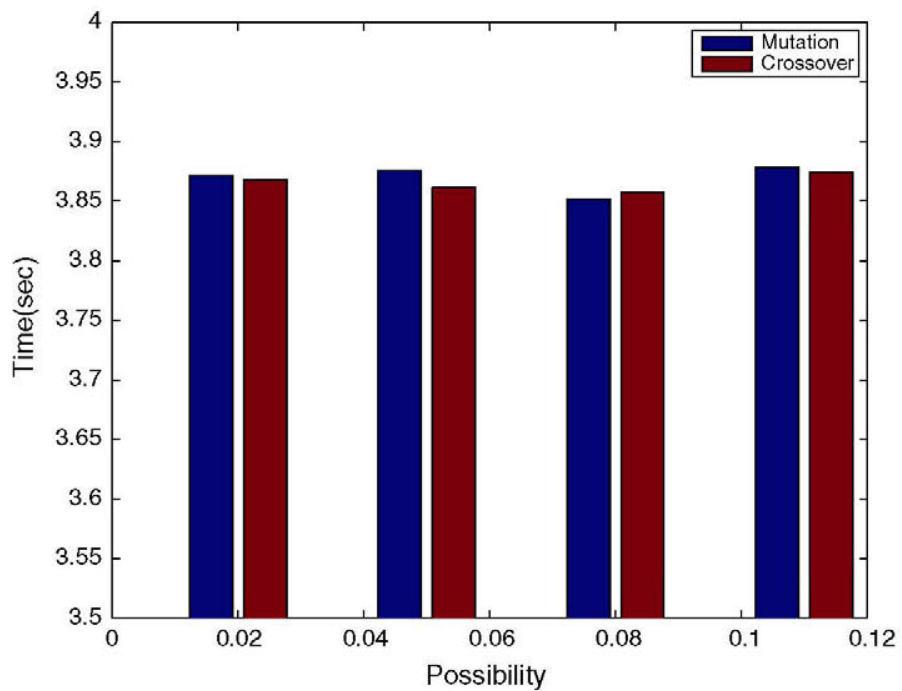

Fig. 15 The impact of mutation and crossover possibilities over desirability

variations do not have significant impact on both the computation time and desirability values.

\subsection{Performance comparison with other heuristics}

Two comparable heuristics (LowCost by Kouakou et al. (2010) and MOGA by Jourdan and de Weck (2004)) are selected and programmed in the same platform as the 
Table 3 Features of algorithms for comparison

\begin{tabular}{llllllllll}
\hline Algorithm & WSN type & Solutions & Radio & \multicolumn{2}{l}{ Objectives } & & & \\
\cline { 5 - 8 } & & & & $\mathrm{C}$ & Cost & $\mathrm{L}$ & $P_{l}$ & $P_{d}$ \\
\hline Proposed & SN, RN, BS & Multiple & Ray-tracing(RT) & Y(RT) & Y & Y & Y & Y \\
WMOGA & SN, RN, BS & Single & Ray-tracing(RT) & Y(RT) & Y & Y & Y & Y \\
MOGA & SN, BS & Single & distance & Y(dist.) & N & Y & N & N \\
LowCost & SN, BS & Single & Line-of-Sight(LoS) & Y(LoS) & Y & N & N & N \\
\hline
\end{tabular}

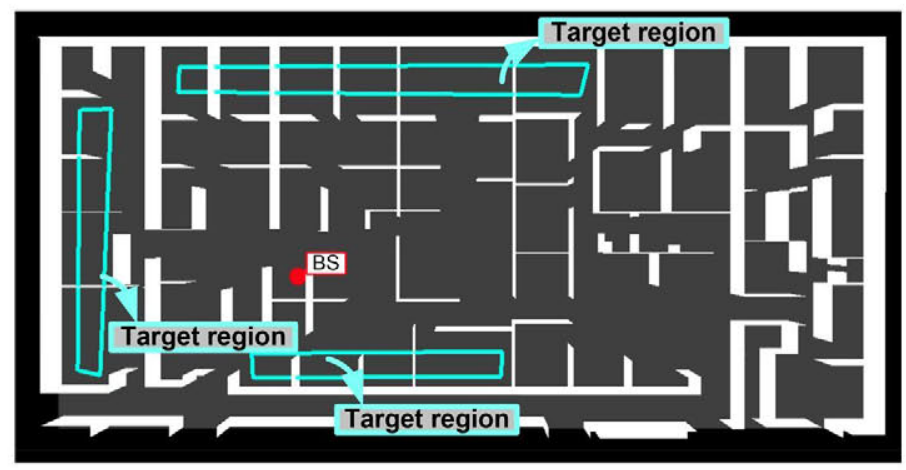

Fig. 16 Scenario East Lansing

proposed algorithm, so that their performance are fairly evaluated in exactly the same configuration. Besides, a weighted multi-objective fitness function (WMOGA) developed in the authors' previous work by He et al. (2013) is modified and implemented to evaluate the impacts of NSGA-II on the final solutions, the formula of the weighted function is:

$$
f=w_{1} D_{C}+w_{2} D_{\text {cost }}+w_{3} D_{L}+w_{4} D_{P_{l}}+w_{5} D_{P_{d}}
$$

Table 3 compares the features of the algorithms, and the proposed method considers more objectives and more practical modelling for heterogeneous WSN. The impact of modelling the radio and sensing signal is not analyzed in this work, however it has been proved in the previous work, the practical ray-tracing algorithm outperforms other distance based empirical models. By setting reflection and diffraction depth as depth $=0$ for the ray-tracing engine, only direct paths are traced in this evaluation. Therefore the algorithms are compared in time efficiency and optimization performance.

The population of Children is 8 for the first 3 algorithms, and the Parent is 8 for the proposed method, 1 for the other two. $N U M_{\max G e n}=150$ for the first 3 algorithms. Three scenarios are tested for all the algorithms and the configurations are: the first scenario is in the CEI-UPM floor plan, $A_{s}=70$ and BS.location = $(17.52,10.02,1.5 \mathrm{~m}$ ) (Fig. 10); the second scenario is based on the floor plan of East Lansing, which has a scale of $77.4 \times 36.6 \times 3 \mathrm{~m}^{3}$, the resolution is $1 \mathrm{~m}, A_{s}=232$ and $B$ S.location $=(23.43,13.60,1.5 \mathrm{~m})$ (Fig. 16); outdoor region of Madrid city is 


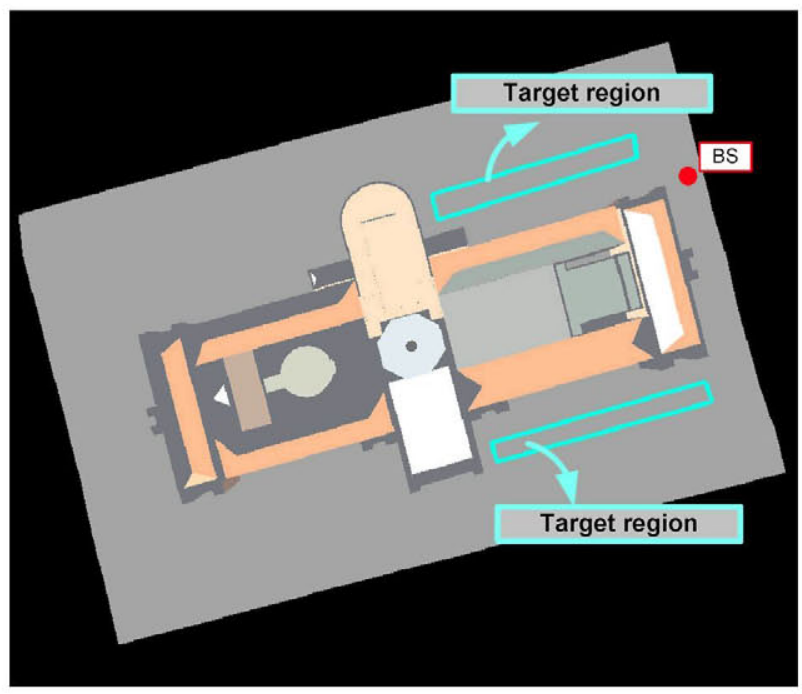

Fig. 17 Scenario Madrid

the third scenario. Its scale is $233.36 \times 297 \times 73.67 \mathrm{~m}^{3}$, resolution is $3 \mathrm{~m}, A_{s}=310$ and BS.location $=(107.8,60.1,3 \mathrm{~m})$ (Fig. 17).

The five desirability metrics used by the proposed method are also computed based on the topologies generated by the compared three algorithms, the solutions are compared according to those values as indicated in Tables 4, 5 and 6 respectively. Italic values the best value and Bold values the worst. The performance of MOGA is limited by using a constant WSN size and unilateral objectives. WMOGA considers all the objectives without the ability of providing multiple solutions simultaneously,

Table 4 Results comparison for Scenario CEI

\begin{tabular}{lllllll}
\hline Algorithm & Time $(s)$ & \multicolumn{2}{l}{ Objectives } & & \\
\cline { 3 - 7 } & & $D_{C}$ & $D_{\text {Cost }}$ & $D_{L}$ & $D_{P_{l}}$ & $D_{P_{d}}$ \\
\hline Proposed & 255.09 & 1 & 0.84 & 0.24 & 0.99 & 0.96 \\
& & 1 & 0.835 & 0.29 & 0.99 & $\mathbf{0 . 8 6}$ \\
& 1 & 0.83 & 0.25 & 0.99 & 1 \\
& & 1 & 0.84 & 0.22 & 0.99 & 0.92 \\
& & 1 & 0.845 & 0.24 & 0.99 & 0.88 \\
& & 1 & 0.84 & 0.22 & 0.99 & 0.99 \\
WMOGA & 456.64 & 1 & 0.85 & 0.26 & 0.99 & 0.98 \\
MOGA & 67.66 & 1 & 0.85 & 0.25 & 0.99 & 1 \\
LowCost & 1.3 & 1 & 0.835 & 0.26 & 0.99 & 0.98 \\
& & 1 & $\mathbf{0 . 8 2}$ & 0.28 & $\mathbf{0 . 9 8}$ & 0.9 \\
& & & $\mathbf{0 . 8 2}$ & $\mathbf{0 . 2 1}$ & 0.99 & 0.96 \\
\hline
\end{tabular}


Table 5 Results comparison for Scenario East Lansing

\begin{tabular}{|c|c|c|c|c|c|c|}
\hline \multirow[t]{2}{*}{ Algorithm } & \multirow[t]{2}{*}{ Time $(s)$} & \multicolumn{5}{|c|}{ Objectives } \\
\hline & & $D_{C}$ & $D_{\text {Cost }}$ & $D_{L}$ & $D_{P_{l}}$ & $D_{P_{d}}$ \\
\hline \multirow[t]{8}{*}{ Proposed } & \multirow[t]{8}{*}{799.32} & 1 & 0.805 & 0.26 & 0.99 & 0.91 \\
\hline & & 1 & 0.81 & 0.24 & 0.99 & 0.97 \\
\hline & & 1 & 0.83 & 0.21 & 0.99 & 0.94 \\
\hline & & 1 & 0.81 & 0.26 & 0.99 & 0.92 \\
\hline & & 1 & 0.815 & 0.30 & 0.99 & 0.96 \\
\hline & & 1 & 0.815 & 0.30 & 0.99 & 0.87 \\
\hline & & 1 & 0.82 & 0.21 & 0.99 & 0.89 \\
\hline & & 1 & 0.815 & 0.32 & 0.99 & 1 \\
\hline WMOGA & 581.03 & 1 & 0.815 & 0.36 & 0.99 & 1 \\
\hline MOGA & 177.14 & 1 & 0.82 & 0.28 & 0.99 & 0.94 \\
\hline LowCost & 3.64 & 1 & 0.82 & 0.19 & 0.99 & 0.96 \\
\hline
\end{tabular}

Table 6 Results comparison for Scenario Madrid

\begin{tabular}{lllllll}
\hline Algorithm & Time (s) & \multicolumn{2}{l}{ Objectives } & & \\
\cline { 3 - 7 } & & $D_{C}$ & $D_{\text {Cost }}$ & $D_{L}$ & $D_{P_{l}}$ & $D_{P_{d}}$ \\
\hline Proposed & \multirow{2}{*}{4048.57} & 1 & 0.83 & 0.19 & 0.99 & 0.95 \\
& & 1 & 0.83 & 0.18 & 0.99 & 0.97 \\
& 1 & 0.83 & 0.20 & 0.99 & 0.95 \\
& & 1 & 0.86 & 0.22 & 0.99 & 0.91 \\
& & 1 & 0.85 & 0.21 & 0.99 & 1 \\
& & 1 & 0.86 & 0.23 & 0.99 & 0.99 \\
& & 1 & 0.83 & 0.20 & 0.99 & 0.94 \\
WMOGA & 1236.32 & $\mathbf{0 . 9 9}$ & 0.755 & 0.18 & 0.99 & 1 \\
MOGA & 1581.14 & 1 & $\mathbf{0 . 5 8}$ & 0.17 & 0.99 & 0.99 \\
LowCost & 35.29 & 1 & $\mathbf{0 . 5 8}$ & $\mathbf{0 . 0 5}$ & 0.99 & $\mathbf{0 . 8 3}$ \\
\hline
\end{tabular}

which explains why this method sometimes obtains good optimization performance. As expected, most of the best results are obtained by the proposed algorithm, allowing designers making decisions from different aspects to construct a reliable topology.

The computation time of all the algorithms increases as the scale of scenario grows. LowCost is the most efficient with deterministic heuristic while it obtains worst performance on some objectives at each scenario. Especially when there are significant obstacles between the unconnected and connected part of a WSN, more nodes are placed across the obstacles instead of placing around them to optimize the cost. The proposed method provides eight different multi-objective optimized solutions simultaneously and leads to the highest time consumption at each scenario. However if 
divided by 8 , the average time consumption per solution is at least $45 \%$ better than MOGA and at least $60 \%$ better than WMOGA. As a result, even by integrating external WSNet simulation in the loop of evolutionary strategy and with changeable size of WSN, the proposed method is still much more efficient than MOGA and WMOGA.

\section{Real measurements and results analysis}

Two environment monitoring demonstrations were set up to validate the performance of the proposed planning algorithm. 'Cookies' sensor nodes developed by Portilla et al. (2006) from CEI-UPM are used in both deployments and measurements. 'Cookie' is designed as a modular architecture of four layers as shown in Fig. 5. In this experiment it is equipped with ZigBee communication protocol layer, an environment sensor that is able to sense temperature, light and humidity, and external antennas made by EAD. The BKR2400 antenna is $1 / 2$ wave dipole with $2 \mathrm{dBi}$ peak gain, it has linear polarisation with omni-directional radiation pattern at horizontal plane. Lithium-based batteries have been used to supply the energy to the modular nodes during the WSN deployments and experimental tests, providing upto $500 \mathrm{mAh}$, which covers the power consumption requirements of the devices. Moreover, they can be charged by using the power supply layer of the Cookie architecture, so that the autonomy of the nodes is enhanced.

\subsection{Aggregation mechanism of measured data}

Besides gathering the ambient data, the most important parameters that should be observed are RSS value of data packets, neighborhood table of each node, routing table with the BS as destination, battery level and packet delivery states. The request of those observations is implemented by means of using the HW-SW co-design platform proposed by Mujica et al. (2012), which is a framework based on libraries and controllers that allows designers realizing applications by programming in $\mathrm{C}$ code and compiling to generate the bitstreams for the microcontroller of sensor node. The mechanism of aggregating the aforementioned data is described as follows:

1. RSS. Whenever a node $N_{i}$ receives packets from other nodes, it records the RSS in $\mathrm{dBm}$, and noted as

$$
R S S\left(N_{j}, t_{k}\right)
$$

Where $N_{j}$ is the source of a packet, $t_{k} \in\left[0, t_{p}\right]$ is the arrival time stamp of the packet, $t_{p}$ is the overall testing time of WSN.

2. Neighborhood table of each node. The neighborhood table $T_{i}$ of $N_{i}$ contains the IDs of neighbors and corresponding RSS records. The format of $T_{i}$ is expressed as following and for simplicity in real application, 10 samples of RSSs are cached for each neighbor of $N_{i}$. 


$$
T_{i}=\left(\begin{array}{c|c}
N_{j_{1}} & R S S I\left(N_{j_{1}}, t_{0}\right), \operatorname{RSSI}\left(N_{j_{1}}, t_{1}\right), \cdots, \operatorname{RSSI}\left(N_{j_{1}}, t_{p}\right) \\
N_{j_{2}} & \operatorname{RSSI}\left(N_{j_{2}}, t_{0}^{\prime}\right), \operatorname{RSSI}\left(N_{j_{2}}, t_{1}^{\prime}\right), \cdots, \operatorname{RSSI}\left(N_{j_{2}}, t_{p}^{\prime}\right) \\
\ddots & \ddots
\end{array}\right)
$$

3. Routing table of the network. It records for the whole WSN the routes from each node to BS. Each node maintains the next hop information, so that at the base station, routing table $R$ of the WSN is constructed (Table 7).

4. Power consumption (Battery level). This observation is to estimate the lifetime of the network. By observing battery level, the battery status of each node can be evaluated. Each node records its battery status $B L$ at every period $t_{B}$ and reports to the $B S$ every $n \cdot t_{B}$. By doing so, the battery level table $B$ of the whole network is expressed as following:

$$
B=\left\langle\begin{array}{c|ccc}
N_{j_{1}} & B L\left(N_{j_{1}}, t_{B}\right), B L\left(N_{j_{1}}, 2 t_{B}\right), \cdots, B L\left(N_{j_{1}}, n t_{B}\right) \\
N_{j_{2}} & B L\left(N_{j_{2}}, t_{B}^{\prime}\right), B L\left(N_{j_{2}}, 2 t_{B}^{\prime}\right), \cdots, B L\left(N_{j_{2}}, n t_{B}^{\prime}\right) \\
\ddots & \ddots &
\end{array}\right\rangle
$$

5. Format of data packet. The BS gathers sensed data from sensors. Each SN periodically $\left(T_{d}\right)$ sends data packet to BS, the data packet contains the following information:

Packet format

\begin{tabular}{lllll}
\hline Source ID & Arrival time stamp & Sequence & Sensed data & TX time stamp \\
\hline
\end{tabular}

Once the test is terminated, the packet loss rate can be analyzed based on the continuity of packet sequence for each node, and packet delay of the WSN is computed from the differences between TX time stamp and arrival time stamp. Moreover, environmental monitoring data are obtained as well to prove that those solutions can satisfy the application requirements.

\subsection{Application interface}

A user application is developed to observe data and maintain the functions of nodes. The application interface is programmed by using JAVA with all the aforementioned

Table 7 Routing table format

\begin{tabular}{lll}
\hline Source & Destination & Next hop \\
\hline$N_{i}$ & BS & $N_{k}$ \\
$N_{j}$ & BS & $N_{\vartheta}$ \\
$\ddots$ & BS & $\ddots$ \\
\hline
\end{tabular}




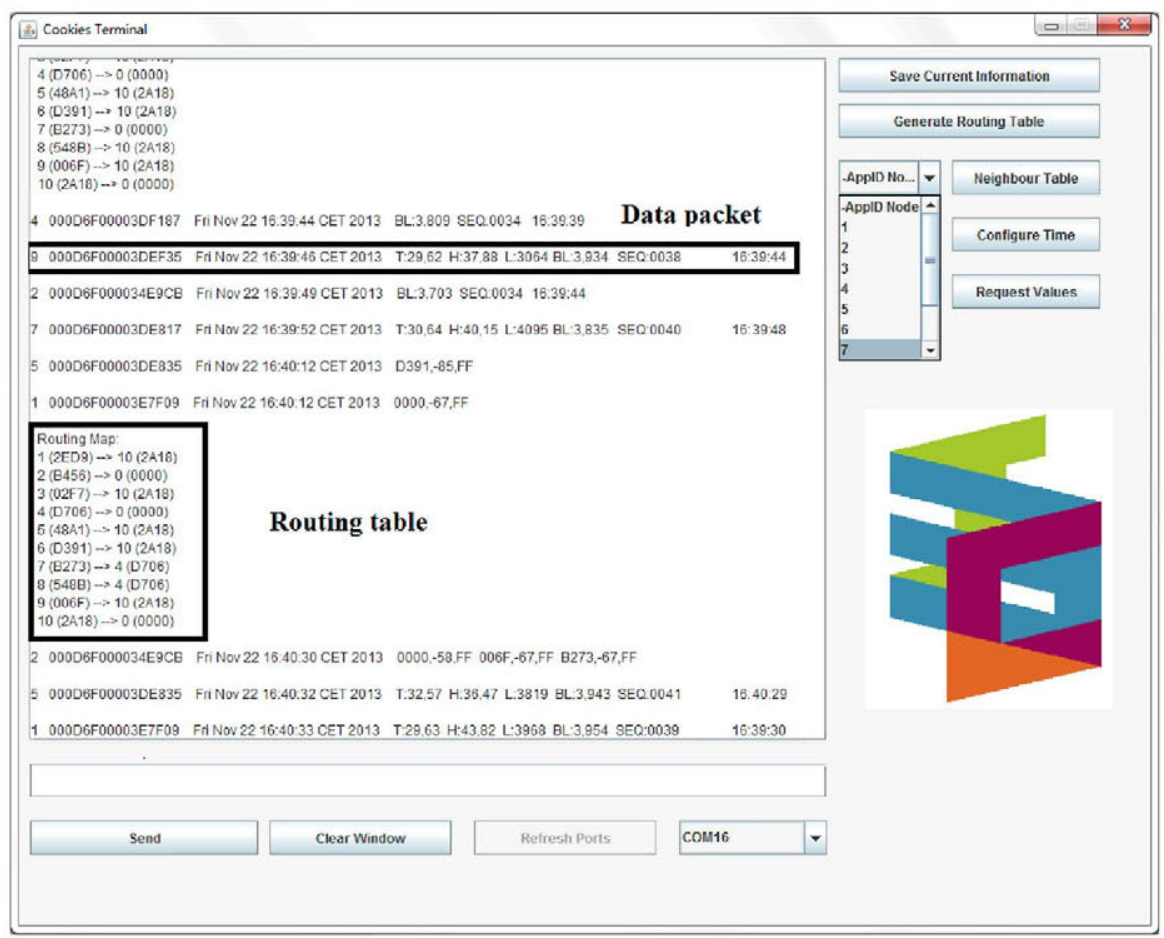

Fig. 18 Application interface

functions to fetch all the required information. It works in cooperation with the BS and Fig. 18 shows the structure of the interface. It allows simple operations from users:

- Data packet is shown once received by BS. As can be seen, the source ID, TX time, temperature(T), humidity (H), light (L), battery level and sequence number are included. Arrival time stamp is added at the end.

- Routing table can be generated according to user's command, which is realized by clicking 'Generate Routing Table' button.

- User can select node ID and click the 'Neighbor Table' to observe the neighborhood table of a node.

- Time is synchronized by clicking 'Configure Time'.

- All the information is saved to '.txt' file once 'Save Current Information' button is pressed.

After preparing all the aforementioned hardware devices, aggregation mechanism and user interface software are used to monitor the WSN. Two real deployments are launched at an indoor and an outdoor environment to monitor temperature, humidity and light level, and validate the planned topologies and estimated performance by the proposed planning algorithm. 


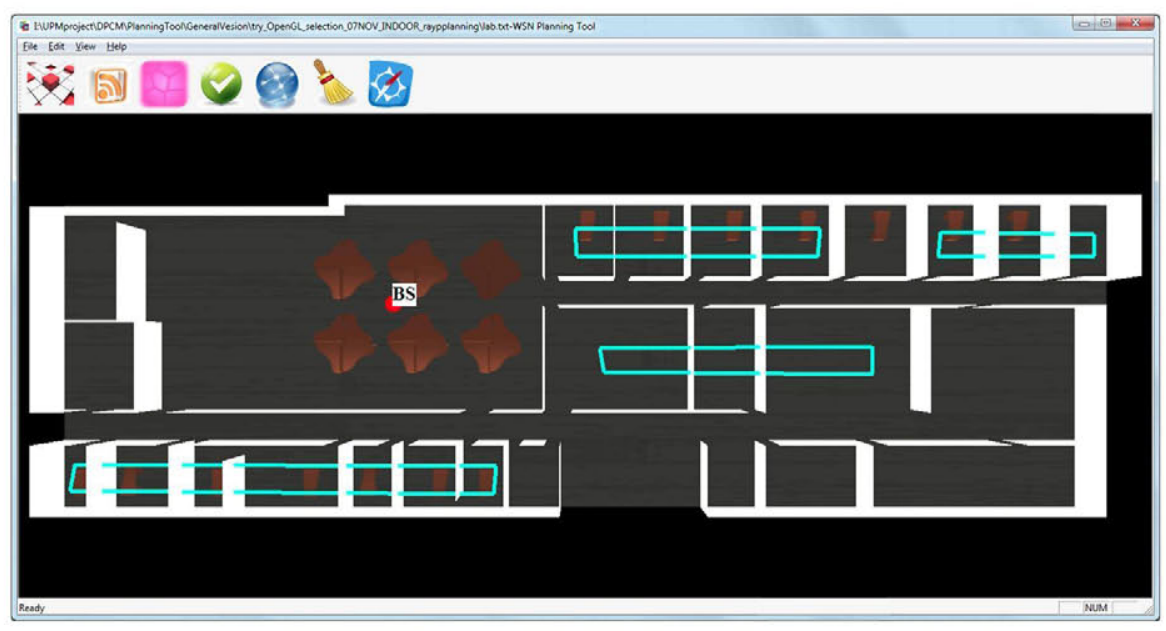

Fig. 19 User requirement over the indoor test

\subsection{Indoor measurements and results analysis}

The first test is realized in the indoor environment of CEI-UPM lab. User requirements on sensing regions are demonstrated in Fig. 19, $A_{s}=90$ and BS.location = $(18.73,12.00,1.5 \mathrm{~m})$. Nodes are set with TX power as $-12 \mathrm{dBm}$, RX sensitivity as $-98 \mathrm{dBm}$ and $R_{\text {sense }}=3 \mathrm{~m}$.

A population of planed topologies are generated by the proposed algorithm with optimized coverage, cost, lifetime, packet latency and packet drop rate. In this work, two candidates, which have topologies indicated in Figs. 20(a) and 21(a), are selected among others for real deployments. All the nodes are placed approximately at the locations indicated by the planned solutions and the constructed topologies are shown in Figs. 20(b) and 21(b) respectively. The routing from $N_{5}$ to BS in the planned solution is via $N_{4}$ whereas it routes though $N_{8}$ in the real deployments. Except $N_{5}$, the remaining nodes have the same next hop as indicated by the planned solutions.

The evaluated performance of the two candidate solutions are shown in Table 8 , topology 1 performs better than topology 2 in terms of cost, lifetime and packet drop rate.

Table 9 shows the measured data of topology 1 in details. The detected neighbors and corresponding RSS values are shown (the first sub-row) for each node $\left(N_{1}-N_{17}\right)$ in the scenario. All the detected RSS values are computed by averaging the fetched samples along different time, and they are compared with the simulation results, by setting the ray-tracing engine with maximum reflection depth depth $=0$ (the second sub-row) and depth $=3$ (the third sub-row). The number of traced rays grows as depth increases, however the results will be more accurate as more multi-path effects are considered.

As can be seen, there are some errors in discovered neighbors of some nodes (e. g. $N_{2}$ and $N_{14}$ ), and the errors are marked by red color. Assuming that $N(u)$ is the set of actual neighbors of a node $u$, and $N^{\prime}(u)$ the set of neighbors known to $u$ (i.e. 


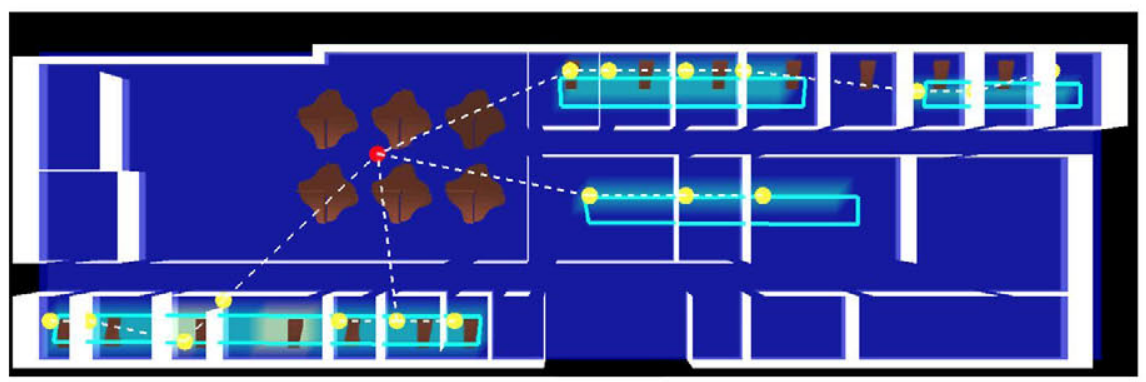

(a) Planned solution by proposed approach

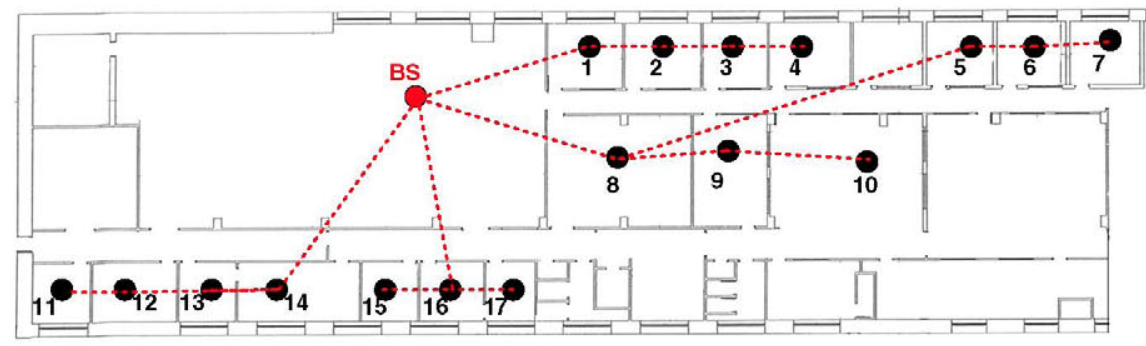

(b) Real topology

Fig. 20 Topology comparison 1: a One of the eight solutions generated for CEI-UPM indoor measurement. b The topology of real deployment

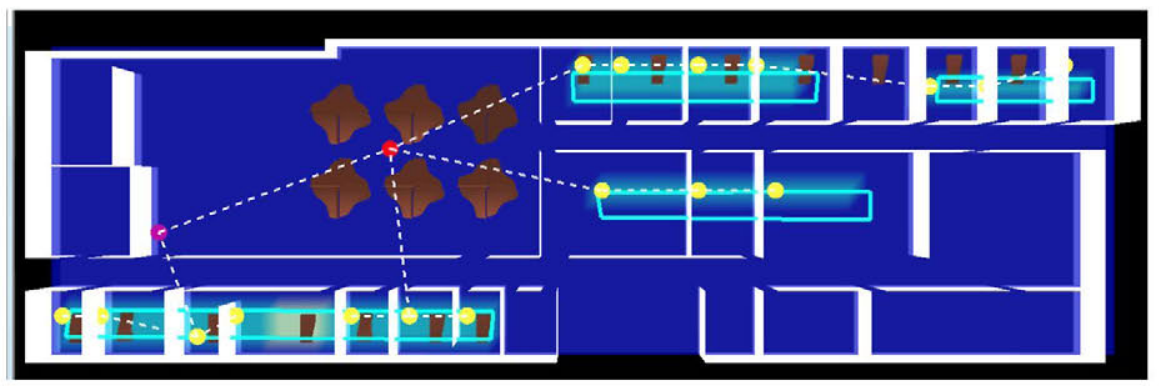

(a) Planned solution by proposed approach

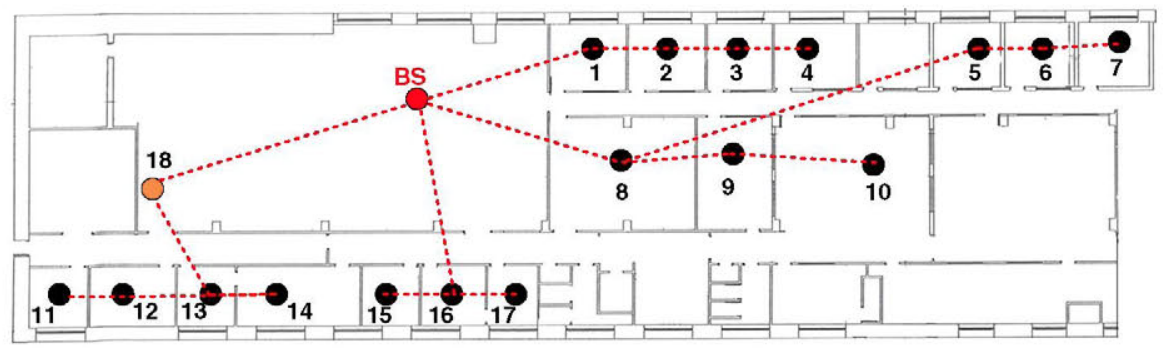

(b) Real topology

Fig. 21 Topology comparison 2: a Another solution generated for CEI-UPM indoor measurement. b The topology of the corresponding real deployment 
Table 8 Evaluated performance of the two candidates

\begin{tabular}{llllll}
\hline \multirow{2}{*}{ Topology solution } & \multicolumn{4}{l}{ Objectives } \\
\cline { 2 - 6 } & $D_{C}$ & $D_{\text {Cost }}$ & $D_{L}$ & $D_{P_{l}}$ & $D_{P_{d}}$ \\
\hline 1 & 1 & 0.83 & 0.432 & 0.99 & 0.99 \\
2 & 1 & 0.825 & 0.428 & 0.99 & 0.95 \\
\hline
\end{tabular}

whose identifier is present in its neighborhood table). Neighborhood accuracy $A c c_{n b}$ is the average value of the accumulation for all the nodes in the WSN the proportion of actual neighbors of node that have been indeed detected. It is formulated as (17):

$$
A c c_{n b}=\frac{\sum_{u=1}^{M} \frac{\left|N(u) \cap N^{\prime}(u)\right|}{\left|N^{\prime}(u)\right|}}{M} \times 100 \% .
$$

Accordingly the accuracy of neighborhood $A c c_{n b}=88 \%$ in this case. The main reasons of this error are from the embedded HELLO protocol of the ZigBee layer. As $N_{2}$ cannot detect $N_{7}$ and $N_{8}$ while $N_{7}$ and $N_{8}$ is able to discover $N_{2}$ as their neighbor, the similar reason can be applied for the neighborhood of $N_{5,6,10,12,13,14,16}$.

Figure 22 demonstrates graphically RSS values of the measured data and simulation results when depth $=0$ and depth $=3$, by aligning the actual neighbors for each node (from $N_{1}$ to $N_{17}$ ). Table 9 shows the mean error $(M E)$ and standard deviation error (STD) for those two simulations. When depth $=0, M E_{0}=4.29 \mathrm{~dB}$ and $S T D_{0}=5.06 \mathrm{~dB}$; If depth $=3, M E_{3}=3.80 \mathrm{~dB}$ and $S T D_{3}=3.61 \mathrm{~dB}$, which as expected, performs better ( $11 \%$ reduction in $M E$ and $28.6 \%$ reduction in $S T D$ ) than the former case. Therefore WSN designers should make a trade-off decision between the time consumption and accuracy, if the planning strategy can be realized without time constraint, this work suggests users configure ray tracing engine with depth $>0$ to improve the accuracy of topology estimation.

This scenario is tested on 22nd and 23rd November, 2013. Sensors detect environment and send data packet every one minute, the battery status is reported from each node to BS every one minute as well. This traffic load mechanism aims to speedup the battery consumption with a ratio of 120 times faster than the simulation period (100 days). Therefore network longevity is predicted as $8.64 \mathrm{~h}$ and the battery should be changed since that moment. The curves of battery consumption shown in Fig. 23 are the measured remaining energy of $N_{1,8,14}$ varied along working time of WSN. According to the test $N_{1}$ has the lowest lifetime around $8.3 \mathrm{~h}$, which obtains $96.1 \%$ of match with the predicted value and indicates a good performance of the proposed work on lifetime modelling.

Figure 24 compares packet delivery status in a period of 30 min for topology 1. $N_{7}$ has the highest packet loss rate $(20 \%)$ because it needs the maximum number of hops to reach BS compared with other nodes. The average packet latency is around $3.5 \mathrm{~s}$ therefore $D_{p_{l}} \approx 0.97$ which is slightly less $(3 \%$ ) than the estimated result. The packet drop rate is calculated as the proportion between lost data packets and total number of packets that have been sent in the WSN. According to the observed packet sequence number for each node, there is $10 \%$ of packet loss in this case, thus the 
Table 9 Neighborhood table and RSS comparisons between real measurement and simulation results: indoor scenario

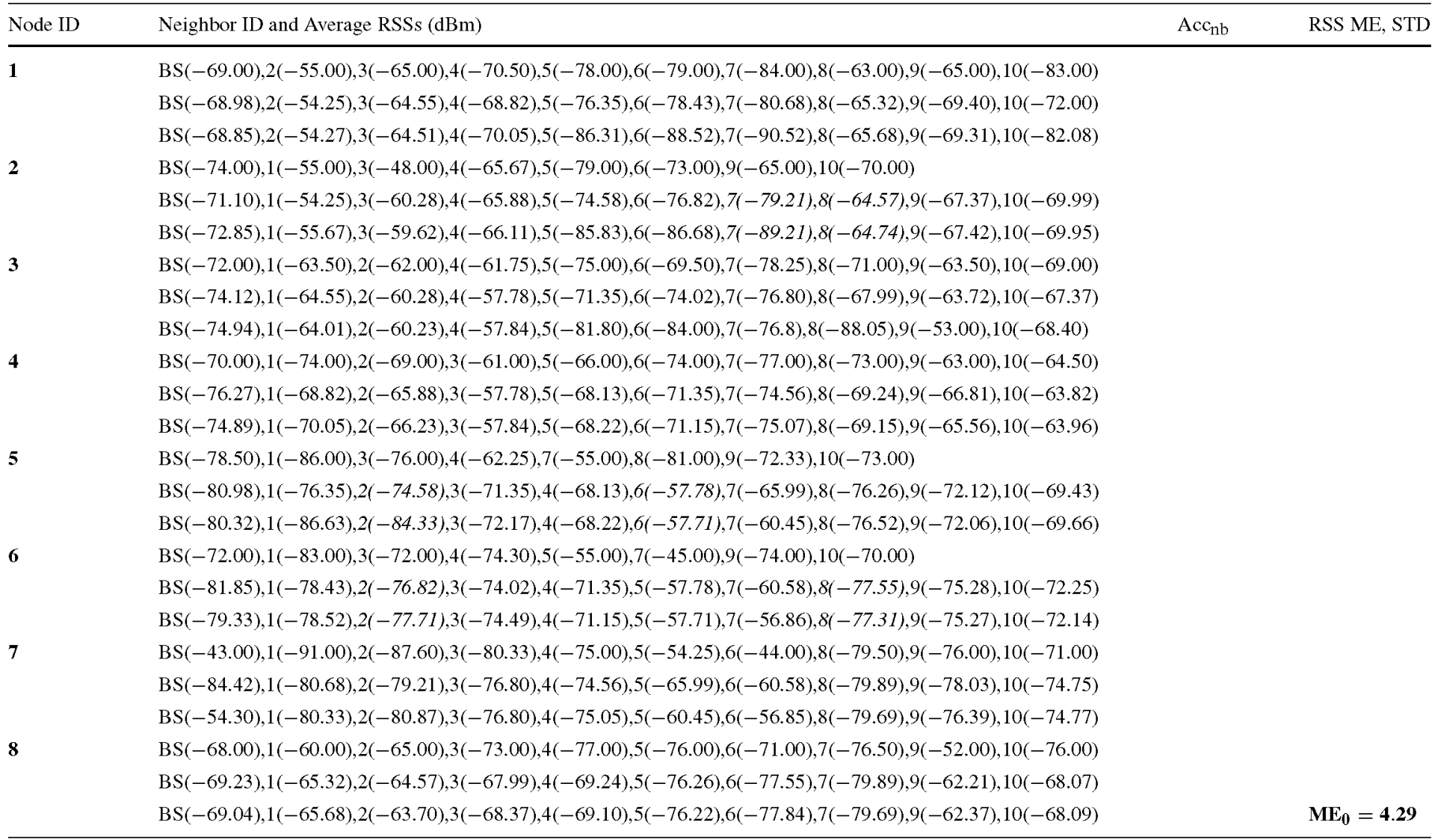


Table 9 continued

Node ID

$11(-73.00), 12(-75.00), 13(-64.00), 15(-56.00), 17(-55.00)$

$B S(-67.03), 11(-76.33), 12(-74.56), 13(-70.60), 14(-68.13), 15(-57.78), 17(-57.78)$

$B S(-66.94), 11(-76.14), 12(-74.72), 13(-69.99), 14(-63.97), 15(-57.10), 17(-57.52)$

17

BS $(-70.00), 11(-77.00), 12(-80.00), 13(-71.00), 14(-72.00), 15(-66.00), 16(-55.00)$

BS $(-69.31), 11(-78.42), 12(-76.80), 13(-73.43), 14(-71.35), 15(-64.55), 16(-57.78)$

BS $(-70.32), 11(-78.89), 12(-77.29), 13(-74.32), 14(-72.24), 15(-64.46), 16(-58.17)$ 


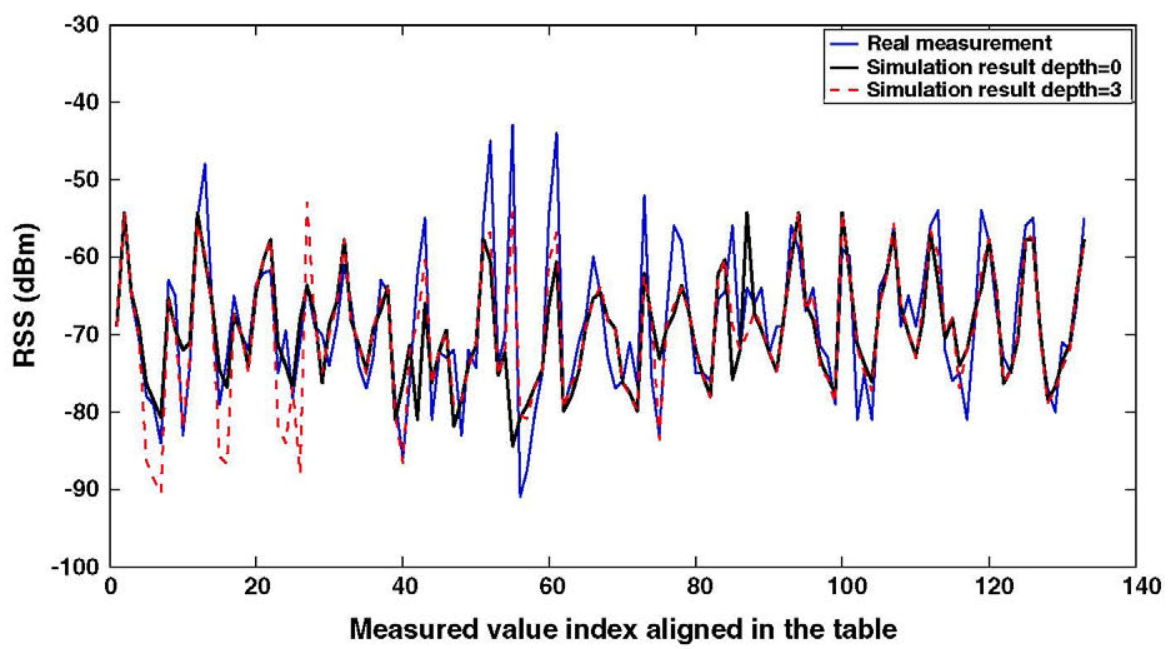

Fig. 22 RSS comparison between real measurement and simulation result with depth $=0$ and depth $=3$

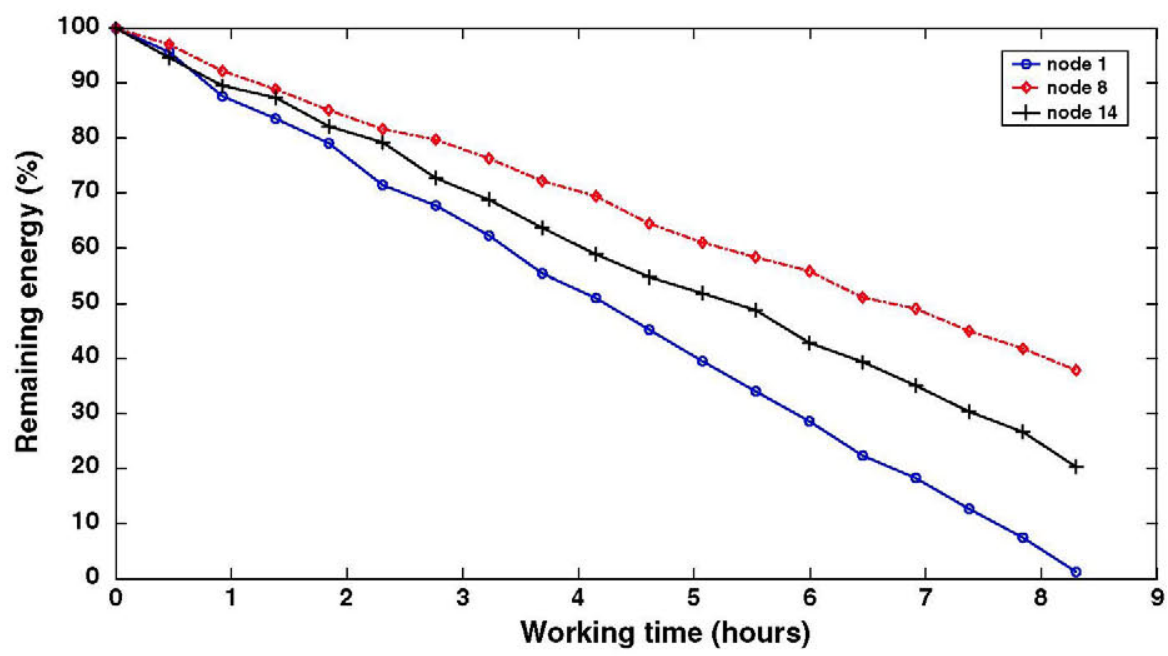

Fig. 23 Remaining energy of $N_{1}, N_{8}$ and $N_{14}$ along the working time of WSN

desirability metric $D_{P_{d}}=90 \%$. Compared with the estimated result (99\%), the error is approximately $9 \%$.

The three nodes: $N_{1,8,14}$ are the bottlenecks of this deployment. Although $N_{1}$ has less children than $N_{8}$ and same as $N_{14}$, the accumulated dropped packets hoping through $N_{1}$ are less than that via $N_{8}$ and $N_{14}$. As a result, $N_{1}$ should forward more data packets to $\mathrm{BS}$ and a faster exhaustion on the battery energy occurs, which also reveals why (see Fig. 23) the lifetime of $N_{1}$ is shorter than other nodes.

Sensed data are gathered at the coordinator and the environment variation is recorded for each sensor. Fig. 25 shows the variation of temperature, humidity and 


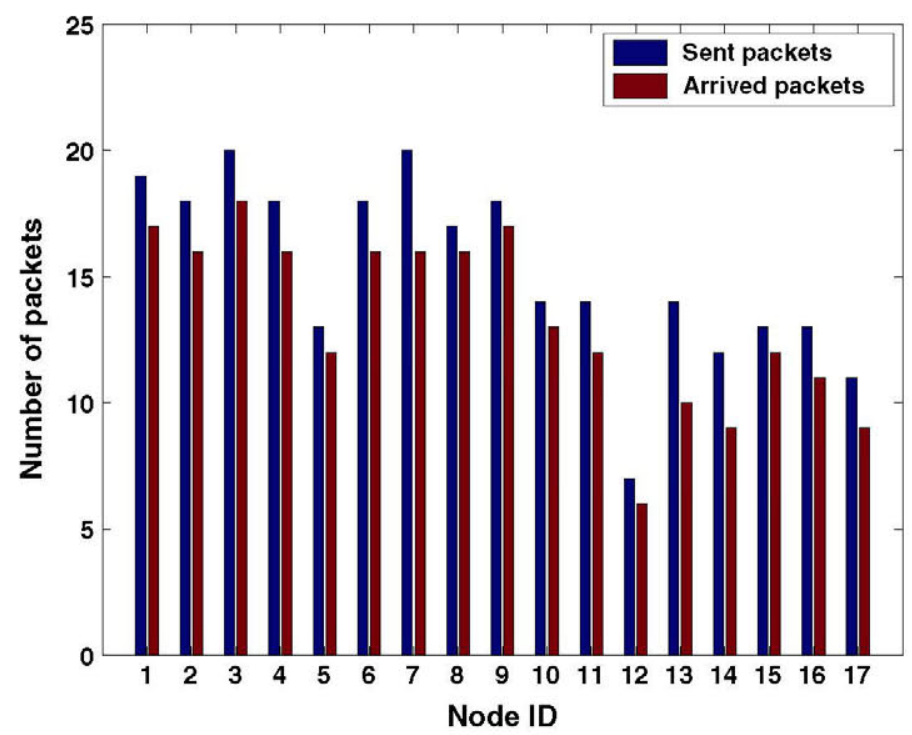

Fig. 24 Compare the number of arrived packet and the number of sent packet for each node in the deployment

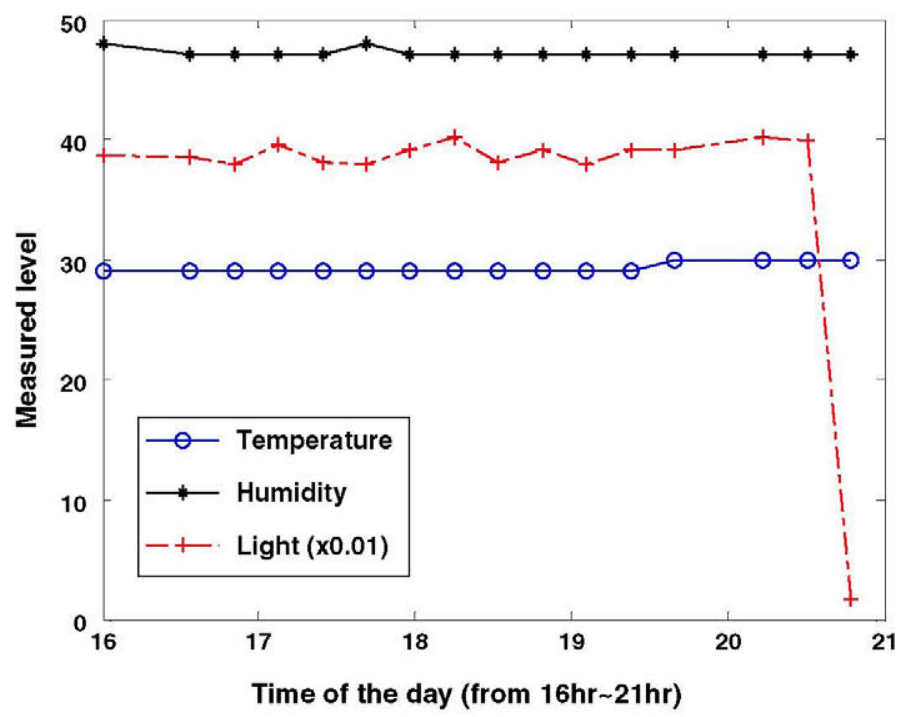

Fig. 25 The sensed data of $N_{4}$

light in the room where $N_{4}$ is placed, and the observation time is from 16:00 to 21:00 on $22 \mathrm{nd}$ Nov, 2013 . The average temperature is $29.25^{\circ} \mathrm{C}$, humidity is 47.13 without significant changes along time. While the light level suddenly reduces from $90.35 \%$ $(3,700)$ to $0 \%(0)$ at $20: 30$, it indicates that the light is turned off and people may have left room after this moment. 


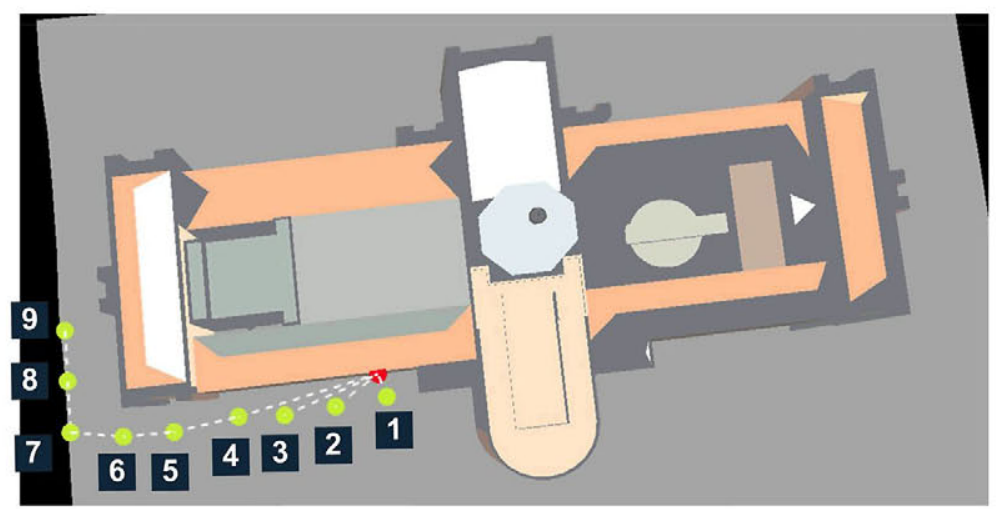

(a) Planned solution with the lowest cost

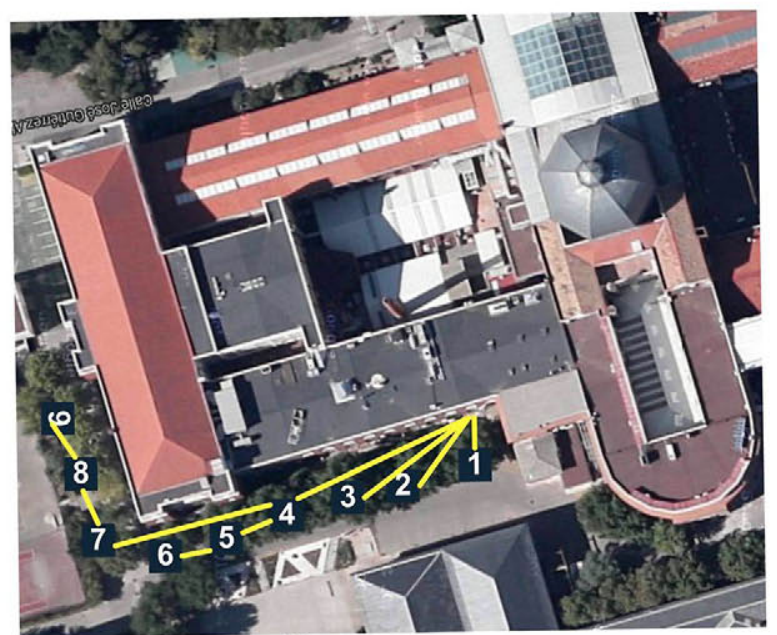

(b) Real topology

Fig. 26 Topology comparison: a One of the eight solutions generated for outdoor environment. b The topology of real deployment

\subsection{Outdoor measurements and results analysis}

The second test is realized to monitor the outdoor environment at the parking lot of UPM. The 3D view of the reconstructed model is shown in (Fig. 17). The scale of this region is $233.36 \times 297 \times 73.67 \mathrm{~m}^{3}$.

User configuration on sensing area for this test is $A_{s}=99$ and TX power for all the nodes are set as $4 \mathrm{dBm}$, which is the maximum communication ability of the antenna. Nodes are set with RX sensitivity as $-98 \mathrm{dBm}, R_{\text {sense }}=8 \mathrm{~m}$. The proposed algorithm generates topology (Fig. 26(a)) accordingly, and the candidate, which with the lowest cost and competitive desirability values of other metrics, is selected in this case. The predicted performance is $D_{C}=1, D_{\text {Cost }}=0.91, D_{L}=0.155, D_{P_{l}}=0.99, D_{P_{d}}=$ 0.98. By placing 9 nodes in target locations, the real topology is shown in Fig. 26(b). 
Table 10 Neighborhood table and RSS comparisons between real measurement and simulation results: outdoor scenario

\begin{tabular}{|c|c|c|c|}
\hline Node ID & Neighbor ID and Average RSSs (dBm) & $A c c_{n b}$ & RSS ME, STD \\
\hline 1 & $\begin{array}{l}\mathrm{BS}(-61.00), 2(-61.50), 3(-67.00), 4(-75.00), 5(-70.00), 6(-79.00), 7(-78.00), 8(-80.00) \\
\mathrm{BS}(-65.65), 2(-62.26), 3(-68.25), 4(-71.40), 5(-74.47), 6(-76.41), 7(-77.99), 8(-81.13)\end{array}$ & & \\
\hline 2 & $\begin{array}{l}\mathrm{BS}(-77.00), 1(-62.30), 3(-58.00), 4(-68.00), 5(-76.00), 6(-74.00), 7(-76.00), 8(-75.00), 9(-86.00) \\
\mathrm{BS}(-72.45), 1(-62.25), 3(-62.19), 4(-67.69), 5(-75.17), 6(-74.53), 7(-76.29), 8(-78.85), 9(-88.02)\end{array}$ & & \\
\hline 3 & $\begin{array}{l}\mathrm{BS}(-57.00), 1(-68.50), 2(-59.00), 4(-62.65), 5(-66.00), 6(-71.00), 7(-75.00), 8(-73.00), 9(-86.00) \\
\mathrm{BS}(-68.11), 1(-68.24), 2(-62.20), 4(-61.15), 5(-68.86), 6(-72.11), 7(-74.51), 8(-72.31), 9(-80.15)\end{array}$ & & \\
\hline 4 & $\begin{array}{l}\mathrm{BS}(-79.00), 1(-74.00), 2(-67.00), 3(-63.00), 5(-70.00), 6(-71.00), 7(-70.00), 8(-77.00), 9(-85.00) \\
\mathrm{BS}(-71.18), 1(-71.44), 2(-67.69), 3(-61.17), 5(-64.31), 6(-69.24), 7(-72.45), 8(-84.86), 9(-85.83)\end{array}$ & & $\mathrm{ME}_{3}=\mathbf{2 . 4 5}$ \\
\hline 5 & $\begin{array}{l}\mathrm{BS}(-73.00), 1(-75.50), 2(-73.00), 3(-67.00), 4(-68.00), 6(-63.00), 7(-69.00), 9(-83.00) \\
\mathrm{BS}(-74.42), 1(-74.57), 2(-72.19), 3(-68.87), 4(-64.31), 6(-61.96), 7(-68.21), 8(-69.44), 9(-83.56)\end{array}$ & $99 \%$ & $\mathrm{STD}_{3}=2.45$ \\
\hline 6 & $\begin{array}{l}\mathrm{BS}(-77.00), 1(-81.00), 2(-74.00), 3(-69.00), 4(-70.33), 5(-65.00), 7(-60.00), 8(-64.00), 9(-78.00) \\
\mathrm{BS}(-76.27), 1(-76.39), 2(-74.51), 3(-72.11), 4(-69.24), 5(-61.99), 7(-62.44), 8(-66.05), 9(-79.89)\end{array}$ & & \\
\hline 7 & $\begin{array}{l}\mathrm{BS}(-79.00), 1(-76.00), 2(-72.00), 3(-76.00), 4(-67.00), 5(-69.00), 6(-59.00), 8(-63.00), 9(-63.00) \\
\mathrm{BS}(-77.81), 1(-77.96), 2(-76.42), 3(-74.55), 4(-72.46), 5(-68.21), 6(-62.45), 8(-62.08), 9(-68.10)\end{array}$ & & \\
\hline 8 & $\begin{array}{l}1(-80.00), 2(-76.00), 3(-78.00), 4(-86.00), 6(-65.00), 7(-61.00), 9(-61.00) \\
1(-80.95), 2(-88.60), 3(-73.21), 4(-84.86), 5(-69.44), 6(-65.95), 7(-62.09), 9(-62.07)\end{array}$ & & \\
\hline 9 & $\begin{array}{l}2(-85.00), 3(-79.00), 4(-85.00), 5(-82.00), 6(-77.00), 7(-67.00), 8(-61.00) \\
2(-88.21), 3(-80.23), 4(-85.83), 5(-83.56), 6(-69.72), 7(-68.09), 8(-62.08)\end{array}$ & & \\
\hline
\end{tabular}




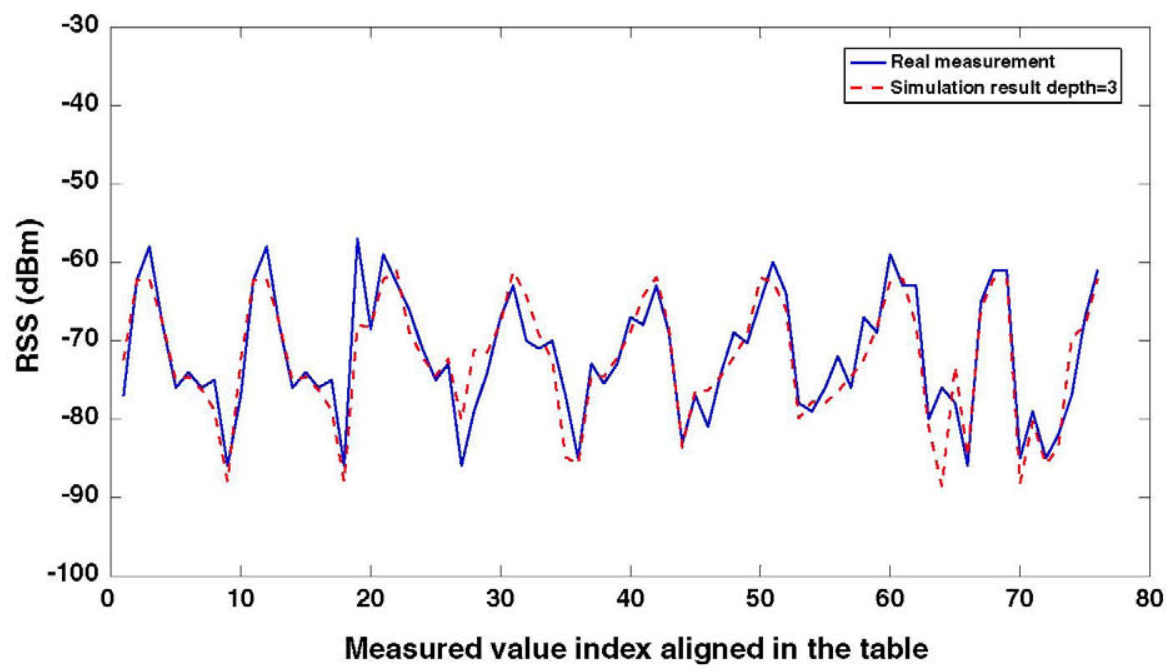

Fig. 27 RSS comparison between real measurement and simulation result with depth $=3$

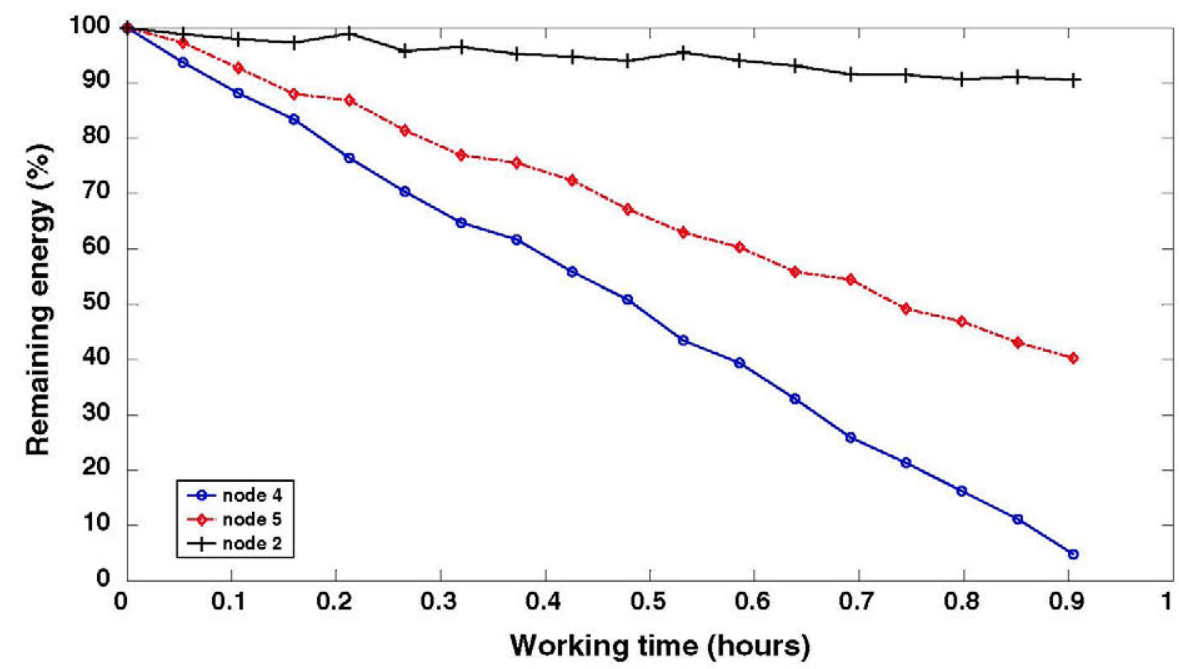

Fig. 28 Remaining energy of $N_{4}, N_{5}$ and $N_{2}$ along the working time of WSN

$N_{1} \sim N_{4}$ are, as estimated, directly connected with BS, $N_{7}$ routes via $N_{4}$ rather than through $N_{6}$ in the solution. Table 10 shows the measured data of the deployed topology in details. All the detected RSS values are computed by averaging the fetched samples along different time, and they are compared with the simulation results with depth $=3$ (the second sub-row). $N_{5}$ and $N_{8}$ can not discover each other in the real deployment while the simulated result shows connections between them, and therefore $A c c_{n b}=98 \%$. Figure 27 demonstrates graphically RSS values of the measured data and simulation results when depth $=3$, by aligning the actual neighbors for each node (from $N_{1}$ to $N_{9}$ ). Table 10 indicates that in this case $M E_{3}=2.45 \mathrm{~dB}$ and 


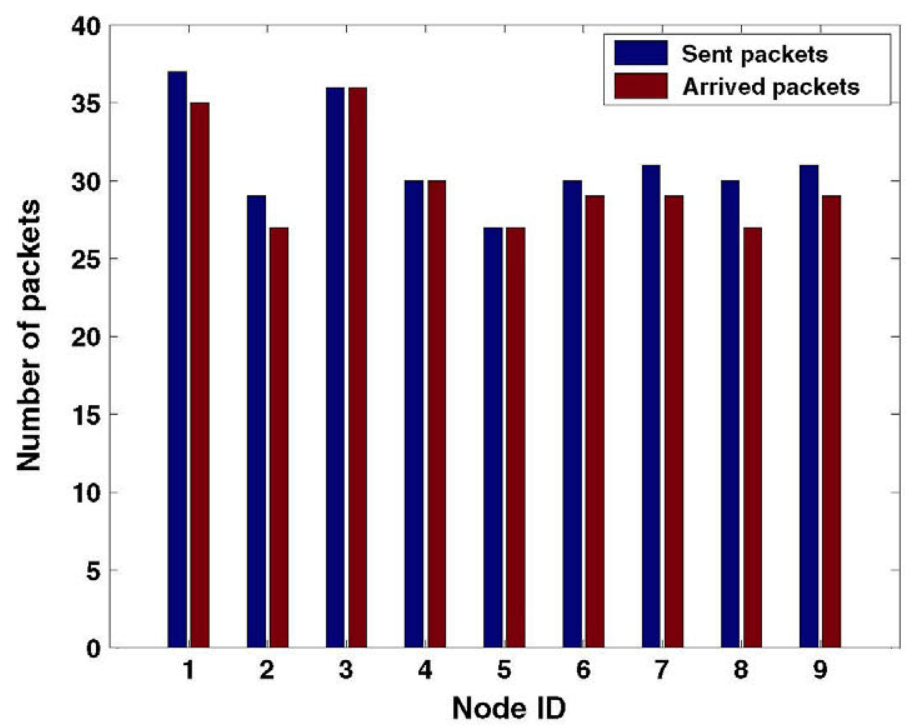

Fig. 29 Compare the number of arrived packet and the number of sent packet for each node in the deployment

$S T D_{3}=2.45 \mathrm{~dB}$. As the outdoor environment is an open area without significant obstacles between nodes, this performance is slightly better than indoor performance. Both indoor and outdoor neighborhood results indicate that the proposed modelling methods on radio propagation and link establishment are practical and reliable to be applied in real deployments.

This scenario is tested on 23rd November, 2013. Sensing period and battery period are one minute. The estimated network longevity is predicted as $1.86 \mathrm{~h}$ with the maximum transmission power. The curves of battery consumptions shown in Fig. 28 are the measured remaining energy of $N_{4,5,2}$ varying along working time of WSN. According to the test, $N_{4}$ has the lowest lifetime $(1.95 \mathrm{~h}$ ), which is $4.8 \%$ higher than the estimated result. $N_{5}$ is in charge of forwarding packets only for $N_{6}$, therefore within the same time it consumes less energy than $N_{4}$. While $N_{2}$ sends packets only for itself, compared with $N_{4}$ and $N_{5}$, it has the longest battery life.

Figure 29 compares packet delivery status in a period of $0.5 \mathrm{~h}$ for the topology. $D_{P_{d}}=99 \%$, which is better than the estimated result of $98 \% . N_{9}$ and $N_{8}$, which perform similarly as $N_{7}$ in the indoor measurement, have the highest packet loss rate $(10 \%)$, because they also need more number of hops to reach BS compared with other nodes. The average packet latency is around $2.6 \mathrm{~s}$, therefore $D_{p_{l}} \approx 0.97$, which is slightly less ( $2 \%)$ than the estimated result.

\section{Conclusion}

An automatic 3D multi-objective optimization WSN planning algorithm is proposed in this work. More comprehensive metrics (connectivity, coverage, cost, lifetime, packet 
latency and packet drop rate) are modeled practically compared with other works. Especially the 3D ray tracing method is used to model the radio link and sensing signal, which is sensitive to the obstruction of obstacles; network routing table is constructed by using AODV protocol; the network longevity, packet delay and packet drop rate are obtained via simulating practical events in WSNet simulator, which is the first time that a network simulator is used in a planning algorithm. Moreover, the multi-objective optimization methodology is developed to cater to the characteristics of WSNs. The individual length is changeable so that the hardware cost is optimized, meanwhile crossovers and mutations are designed to eliminate invalid modifications to improve the computation efficiency. Results are more comprehensively optimized compared with other state-of-the-art algorithms, and the capability of providing multiple optimized solutions simultaneously allows users making their own decisions.

Real deployments for ambient monitoring are realized for both indoor and outdoor regions. Experiments are carefully designed by programming the sensor nodes coping with the application requirements, and the placements of nodes strictly follow the planned topologies generated by the proposed algorithm. The experimental data are categorized and compared in terms of routing table accuracy, neighborhood table accuracy, RSS accuracy, lifetime and packet delivery status. Both applications show the potential of this proposed algorithm to fulfill user requirements with optimized and practical performance.

In the future, the scalability of the computation and probabilistic model of sensing signal should be tackled, the adaptation of TX power should be included by the optimization algorithm to control power consumption and topology.

Acknowledgments The authors would like to acknowledge the support of ARTEMIS JU and Spanish Ministry of Industry and commerce for WSN DPCM project under grant ART-010000-2011-1.

\section{References}

Akbarzadeh, V., Gagne, C., Parizeau, M., Argany, M., Mostafavi, M.: Probabilistic sensing model for sensor placement optimization based on line-of-sight coverage. IEEE Trans. Instrum. Meas. 62(2), 293-303 (2013). doi:10.1109/TIM.2012.2214952

Beutel, J., Kasten, O., Ringwald, M.: Poster abstract: Btnodes-a distributed platform for sensor nodes. In: Proceedings of the 1st International Conference on Embedded Networked Sensor Systems, ACM Press, Los Angeles, California, USA, pp. 292-293 (2003)

Bluetooth.: https://www.bluetooth.org/ (1998)

Cheng, X., Du, D.Z., Wang, L., Xu, B.: Relay sensor placement in wireless sensor networks. Wirel Netw 14(3), 347-355 (2008). doi:10.1007/s11276-006-0724-8

Deb, K., Pratap, A., Agarwal, S., Meyarivan, T.: A fast and elitist multiobjective genetic algorithm: Nsga-II. IEEE Trans. Evol. Comput. 6(2), 182-197 (2002). doi:10.1109/4235.996017

Efrat, A., Har-Peled, S., Mitchell, J.S.B.: Approximation algorithms for two optimal location problems in sensor networks. In: Proceedings of the 2 nd International Conference on Broadband Networks (BroadNets 2005), vol 1, pp. 714-723 (2005). doi:10.1109/ICBN.2005.1589677

Guinard, A., Aslam, M., Pusceddu, D., Rea, S., McGibney, A., Pesch, D.: Design and deployment tool for in-building wireless sensor networks: A performance discussion. In: IEEE 36th Conference on Local Computer Networks (LCN), pp. 649-656 (2011). doi:10.1109/LCN.2011.6115530

He, D., Liang, G., Portilla, J., Riesgo, T.: A novel method for radio propagation simulation based on automatic 3D environment reconstruction. In: Proceedings of the 6th European Conference Antennas and Propagation (EUCAP), pp. 1445-1449 (2012) 
He, D., Portilla, J., Riesgo, T.: A 3d multi-objective optimization planning algorithm for wireless sensor networks. In: IECON (2013)

Huang, Y.K., Hsiu, P.C., Chu, W.N., Hung, K.C., Pang, A.C., Kuo, T.W., Di, M., Fang, H.W.: An integrated deployment tool for zigbee-based wireless sensor networks. In: IEEE/IFIP International Conference on Embedded and Ubiquitous Computing, 2008. EUC '08, vol 1, pp. 309-315, 2008, doi:10.1109/EUC. 2008.87

Johnson, D., Hu, Y., Maltz, D.: The dynamic source routing protocol (DSR) for mobile ad hoc networks for ipv4 (2007)

Jourdan, D., de Weck, O.: Layout optimization for a wireless sensor network using a multi-objective genetic algorithm. In: Vehicular Technology Conference, 2004. VTC 2004-Spring. 2004 IEEE 59th, vol 5, pp. 2466-2470 (2004). doi:10.1109/VETECS.2004.1391366

Kim, S., Ko, J.G., Yoon, J., Lee, H.: Multiple-objective metric for placing multiple base stations in wireless sensor networks. In: 2nd International Symposium on Wireless Pervasive Computing, 2007. ISWPC'07, 2007, doi:10.1109/ISWPC.2007.342679

Kling, R.M.: Intel mote: An enhanced sensor network node. In: Proceedings of the International Workshop on Advanced Sensors, Structural Health Monitoring, and Smart Structures, Raiosha, Japan, pp. 1-6 (2003)

Langendoen, K., Baggio, A., Visser, O.: Murphy loves potatoes: experiences from a pilot sensor network deployment in precision agriculture. In: Proceedings of the 20th international conference on Parallel and distributed processing, IEEE Computer Society, Washington, DC, USA, IPDPS'06, pp, 174-174 (2006)

Lau, S.Y., Chang, T.H., Hu, S.Y., Huang, H.J., de Shyu, L., Chiu, C.M., Huang, P.: Sensor networks for everyday use: the bl-live experience. In: IEEE International Conference on Sensor Networks, Ubiquitous, and Trustworthy Computing, 2006, vol 1, pp. 7, 2006, doi:10.1109/SUTC.2006.1636198

Lee, S., Lee, M.: Qrmsc: Efficient qos-aware relay node placement in wireless sensor networks using minimum steiner tree on the convex hull. In: International Conference on Information Networking (ICOIN), 2013, pp. 36-41, 2013, doi:10.1109/ICOIN.2013.6496348

Liu, L., Ma, H.: On coverage of wireless sensor networks for rolling terrains. IEEE Trans. Parallel Distrib. Syst. 23(1), 118-125 (2012). doi:10.1109/TPDS.2011.69

Kouakou, M.T., Yamamoto, S., Yasumoto, K., Ito, M.: Cost-efficient deployment for full-coverage and connectivity in indoor 3d wsns. In: Proceedings of the IPSJ Dicomo 2010 (2010)

Mafuta, M., Zennaro, M., Bagula, A., Ault, G., Gombachika, H., Chadza, T.: Successful deployment of a wireless sensor network for precision agriculture in malawi. In: IEEE 3rd International Conference on Networked Embedded Systems for Every Application (NESEA), 2012, pp. 1-7, 2012, doi:10.1109/ NESEA.2012.6474009

McGibney, A., Guinard, A., Pesch, D.: Wi-design: A modelling and optimization tool for wireless embedded systems in buildings. In: IEEE 36th Conference on Local Computer Networks (LCN), 2011, pp. 640-648, 2011, doi:10.1109/LCN.2011.6115529

Misra, S., Hong, S.D., Xue, G., Tang, J.: Constrained relay node placement in wireless sensor networks: formulation and approximations. IEEE/ACM Trans. Netw. 18(2), 434-447 (2010). doi:10.1109/TNET. 2009.2033273

Mujica, G., Rosello, V., Portilla, J., Riesgo, T.: Hardware-software integration platform for a wsn testbed based on cookies nodes. In: IECON 2012-38th Annual Conference on IEEE Industrial Electronics Society, pp. 6013-6018, 2012, doi:10.1109/IECON.2012.6389099

Perkins, C., Belding-Royer, E., Das, S.: Ad hoc on-demand distance vector (AODV) routing (2003)

Poduri, S., Pattem, S., Krishnamachari, B., Sukhatme, G.S.: Sensor network configuration and the curse of dimensionality (2006)

Polastre, J., Szewczyk, R., Culler, D.: Telos: enabling ultra-low power wireless research. In: Fourth International Symposium on Information Processing in Sensor Networks, 2005. IPSN 2005, pp. 364-369, 2005, doi:10.1109/IPSN.2005.1440950

Portilla, J., de Castro, A., de la Torre, E., Riesgo, T.: A modular architecture for nodes in wireless sensor networks. J. UCS 12(3), 328-339 (2006)

Romer, K., Mattern, F.: The design space of wireless sensor networks. IEEE Wirel. Commun. 11(6), 54-61 (2004). doi:10.1109/MWC.2004.1368897

Shams, S., Chowdhury, A., Kim, K.H., Lee, N.B.: A fast approximation algorithm for relay node placement in double-tiered wireless sensor network. In: Military Communications Conference, 2008. MILCOM 2008. IEEE, pp. 1-6, 2008, doi:10.1109/MILCOM.2008.4753648

Tyndall.: Tyndall mote. http://www.tyndall.ie/content/wireless-sensor-networks (2013) 
UWB.: Iso/iec 26907:2009 information technology-telecommunications and information exchange between systems-high-rate ultra-wideband phy and mac standard (2009)

Waspmote.: Waspmote-wireless sensor networks 802.15.4 zigbee mote. http://www.libelium.com/ products/waspmote (2013)

Xiong, S., Yu, L., Shen, H., Wang, C., Lu, W.: Efficient algorithms for sensor deployment and routing in sensor networks for network-structured environment monitoring. In: INFOCOM, 2012 Proceedings IEEE, pp. 1008-1016, 2012, doi:10.1109/INFCOM.2012.6195455

ZigBee.: http://www.zigbee.org/ (2007) 